\title{
Rheological Properties of Hydrophobically Associative Copolymers Prepared in a Mixed Micellar Method Based on Methacryloxyethyl-dimethyl Cetyl Ammonium Chloride as Surfmer
}

\author{
Rui Liu, ${ }^{1,2}$ WanFen Pu, ${ }^{1,2}$ Hu Jia, ${ }^{1,2}$ XiaoPei Shang, ${ }^{2}$ Yue Pan, ${ }^{3}$ and ZhaoPeng Yan ${ }^{2}$ \\ ${ }^{1}$ State Key Laboratory of Oil and Gas Reservoir Geology and Exploitation, Southwest Petroleum University, Chengdu 610500, China \\ ${ }^{2}$ School of Petroleum and Gas Engineering, Southwest Petroleum University, Chengdu 610500, China \\ ${ }^{3}$ CNOOC Research Institute, CNOOC Building, Beijing 100027, China
}

Correspondence should be addressed to Rui Liu; breakthroughliu@163.com

Received 18 January 2014; Accepted 11 April 2014; Published 15 May 2014

Academic Editor: Peng He

Copyright (C) 2014 Rui Liu et al. This is an open access article distributed under the Creative Commons Attribution License, which permits unrestricted use, distribution, and reproduction in any medium, provided the original work is properly cited.

\begin{abstract}
A novel cationic surfmer, methacryloxyethyl-dimethyl cetyl ammonium chloride (DMDCC), is synthesized. The micellar properties, including critical micelle concentration and aggregation number, of DMDCC-SDS mixed micelle system are studied using conductivity measurement and a steady-state fluorescence technique. A series of water-soluble associative copolymers with acrylamide and DMDCC are prepared using the mixed micellar polymerization. Compared to conventional micellar polymerization, this new method could not only reasonably adjust the length of the hydrophobic microblock, that is, $N_{H}$, but also sharply reduce the amount of surfactant. Their rheological properties related to hydrophobic microblock and stickers are studied by the combination of steady flow and linear viscoelasticity experiments. The results indicate that both the hydrophobic content and, especially the length of the hydrophobic microblock are the dominating factors effecting the intermolecular hydrophobic association. The presence of salt influences the dynamics of copolymers, resulting in the variation of solution characters. Viscosity measurement indicates that mixed micelles between the copolymer chain and SDS molecules serving as junction bridges for transitional network remarkably enhance the viscosity. Moreover, the microscopic structures of copolymers at different experimental conditions are conducted by ESEM. This method gives us an insight into the preparation of hydrophobically associative water-soluble copolymers by cationic surfmer-anionic surfactant mixed micellar polymerization with good performance.
\end{abstract}

\section{Introduction}

The incorporation of a few hydrophobic units into a hydrophilic backbone results in hydrophobically associative watersoluble polymers. In aqueous solution, above a certain polymer concentration, the hydrophobic moieties associate and build a transitory three-dimensional cross-links, leading to unique rheological properties such as dramatic viscosity enhancement [1,2], gelation [3], time-dependent effects [4], and marked elasticity $[5,6]$. Such salient features result from the reversible association processes of the physical networks occurring under shear compared to that of polymers without associative groups. These polymers have triggered great attention in various applications, including petrochemicals, cosmetics, and pharmaceuticals [7-9].

There have been substantial efforts with regard to the synthesis and properties of hydrophobically modified watersoluble polyacrylamides. Following Strauss et al. $[10,11]$ in the 1960s who developed the original hydrophobically modified water-soluble polymers called polysoaps by using $n$-dodecyl bromide and decyl vinyl ether as hydrophobic monomers, McCormick et al. [12], Morishima et al. [13], and Winnik et al. [14] launched an extensive research on such polymers from photophysical and rheological viewpoints. Notably, one of the most classical and well-studied methods to copolymerize acrylamide (AM) with a hydrophobic comonomer 
is a free radical micellar copolymerization technique [1]. However, the addition of well amount surfactant (above the critical micelle concentration, CMC) caused some negative influences, such as low-molecular weight caused by the chain transfer effects of surfactants [5], impurity within the surfactant, and the complicated treatment to remove the surfactants to obtain pure products [15]. An additional problem originates from the difficulty to prepare copolymers with both high associativity and good water compatibility because the microblocky hydrophobe of the copolymers is very limited to maintain solubility in water [16].

To overcome these drawbacks, the conventional micellar copolymerization method is modified by using polymerizable surfactants, that is, surfmers, to substitute the traditional ones. Surfmers are a family of unique surfactants which not only have amphiphilic structures, that is, cationic or anionic long-chain alkyl structure, but also contain polymerizable vinyl double bonds [17-19]. Surfmer molecules form micelles at concentration above the CMC. Both micellar aggregation $\left(N_{\text {agg }}\right)$ and CMC of such micellar systems could be mediated by adding either cosurfactants, such as electrolytes and medium chain-length alcohols, or surfactants [20-23]. Therefore, the hydrophobic microblock length, that is, $N_{H}$, of the copolymers chains was controlled. Mixing behavior of surfmer and cosurfactant has been widely investigated $[24,25]$. On the other hand, due to an amazing interaction between the surfactants that are mixed, mixed micelles composed of binary surfactants have received increasing attention. Properties of binary mixed micelles upon hydrocarbonhydrocarbon, hydrocarbon-fluorocarbon surfactants including nonionic-nonionic, anionic-nonionic, anionic-cationic, and zwitterionic-anionic mixtures were extensively investigated by advanced analytical techniques such as light scattering, small angle neutron scattering, and fluorescence probing measurement $[26,27]$. Based on these experiments, a regular solution theory that accounts well for the characters of binary mixed micelles has been established in which the CMC values and $N_{\text {agg }}$ values involved in different compositional mixtures depended on an interaction parameter $(\beta)$ [22]. Apparently, the regular solution theory is also applied well in the surfactant-surfmer mixed micelle system. Chang and McCormick [28] firstly suggested that the microblock length of the cationic polymerizable surfactant dimethyl-dodecyl (2acrylamidoethyl) ammonium bromide could be controlled by addition of cetyltrimethyl-ammonium bromide (CTAB) as a cationic cosurfactant. Direct experimental evidence of this behavior is reported by Zhang and Wu [23] from conductivity and fluorescence studies on the same mixed micelle system. Stähler et al. [29] found that cationic fluorocarbonhydrocarbon surfmer pairs that bear the same hydrophobic compound may have the partial compatibility in single type of mixed micelles despite the repulsive headgroup interactions.

It is thought that only the surfmer-surfactant mixed micellar system corresponds to the same charge, either positive or negative, the microblock of length could be adjusted. Can the cationic surfmer-anionic surfactant binary system share the same capacity in the preparation of hydrophobically associative polyacrylamide with good performance? This paper just addressed this issue. Firstly, a long-chain alkyl cationic surfmer of methacryloxy-ethyl-dimethyl cetyl ammonium chloride (DMDCC) was synthesized, and the properties of DMDCC-SDS (sodium dodecyl sulfate) mixed micelles are determined by conductivity measurement and a steady-state fluorescence technique. We discuss the synthesis and characterization of cationic associating copolymers prepared by DMDCC-SDS mixed micellar copolymerization with DMDCC as cationic hydrophobic comonomer. Particularly, we focus on the effect of the length and number of the hydrophobic microstructures on the rheological properties. In addition, we look at the effect of salt and surfactant on the apparent viscosity of copolymers.

\section{Experimental Section}

2.1. Materials. Acrylamide (Chengdu KeLong Chemical Reagent Co., Ltd.) was recrystallized twice from acetone and vacuum dried at room temperature prior to use. The azoinitiator V-50 (2,2' -azobis(2-amidinopropane)dihydrochloride) (UA, Alfa Aesar 99\%) was used without further purification. N,N-Dimethyl-ethylamine methacrylate and cetyl chloride, purchased from Aldrich chemicals (purity, 99\%), were used as received. Water was double deionized with a Millipore Milli-Q system. Other reagents were analytically pure and used as received.

\subsection{Synthesis of Cationic Polymerizable Surfactant (DMDCC).} Scheme 1 illustrates the synthesis of the cationic surfmer, methacryloxyethyl-dimethyl cetyl ammonium chloride (DMDCC). N,N-Dimethylethylamine methacrylate $(28.2 \mathrm{~g}$, $0.2 \mathrm{~mol}$ ) was added into a $250 \mathrm{~mL}$ three-necked roundbottom flask and placed in an oil bath and agitated by a magnetic stirrer under a nitrogen atmosphere. Cetyl chloride $(46.9 \mathrm{~g}, 0.180 \mathrm{~mol}$ ) was subsequently added to the additional funnel. When the temperature increased to $85^{\circ} \mathrm{C}$, cetyl chloride was added to the flask dropwise over a period of $6 \mathrm{~h}$. After $42 \mathrm{~h}$, the yellow viscous gel was obtained. The excess crude product was dissolved in a small amount of acetone at $45^{\circ} \mathrm{C}$ and cooled to $-10^{\circ} \mathrm{C}$ and the yellow semisolid product was obtained. Further purification of the product was accomplished by recrystallization from a $1: 1$ (wt/wt) mixture of acetone and ether, yield of $61.8 \mathrm{~g}$ (85\%).

\subsection{Properties of DMDCC-SDS Binary Surfactant System}

2.3.1. CMC Values. CMC values of DMDCC and mixture of DMDCC-SDS were determined by conductivity on a digit conductivity meter. The conductivity, $\kappa$, of aqueous surfactant solutions was measured against the surfactant molar concentration at $50^{\circ} \mathrm{C}$. The difference in the magnitude of the gradients above or below the inflection points in each sample results in a concomitant increase in the uncertainty of the CMC values. Thus, the CMC values of DMDCC and DMDCC-SDS were measured from the break points of the $\kappa$ to $c$ plots.

2.3.2. Aggregation Number. Using pyrene as a probe, the aggregation numbers $\left(N_{\mathrm{agg}}\right)$ of DMDCC as well as mixture 


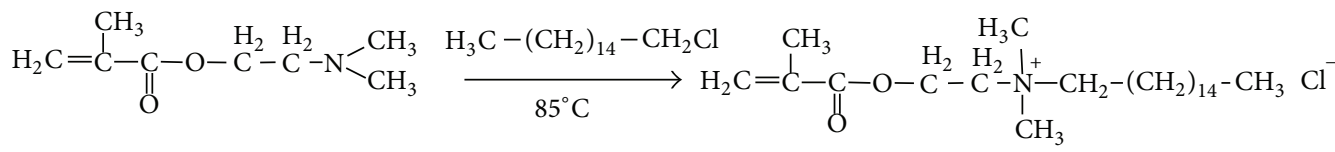

SCHEme 1: Synthesis of the cationic surfmer (DMDCC).

of DMDCC-SDS surfactants at different bulk mole fractions $\alpha_{1}$ were determined by a steady-state fluorescence technique. Samples solutions with $0.1 \mathrm{mM}$ of pyrene were prepared by adding a small amount of concentrated pyrene dissolved in acetone. All the samples were heated at $50^{\circ} \mathrm{C}$ for $12 \mathrm{~h}$ and then filtered with a $0.2 \mu \mathrm{m}$ membrane filter before measurement. The quenching of pyrene monomer fluorescence, originating from excimer formation, was used to determine $N_{\text {agg }}$. Fluorescence decay data corresponded to the following equation:

$$
\ln \left(\frac{I_{0}}{I}\right)=N \frac{[Q]}{\left(C_{t}-\mathrm{CMC}\right)},
$$

where $I_{0}$ and $I$ are the fluorescence intensities in the absence and presence of quencher of the pure/binary surfactant systems. [Q], $C_{t}$, and CMC are the quencher concentration, total surfactant concentration, and critical micelle concentration, respectively. The $\ln \left(I_{0} / I\right)$ to [Q] at different compositions is pseudo-first-order. Therefore, values of Nagg were calculated from the slope of the pseudolinear fits. In each case the total surfactant concentration was constant.

2.4. Synthesis of the Copolymers. A $250 \mathrm{~mL}$ three-necked round-bottom flask is equipped with a mechanical stirrer, nitrogen inlet, and a thermometer. Acrylamide (AM) and methacryloxyethyl-dimethyl cetyl ammonium chloride (DMDCC) and sodium dodecyl sulfate (SDS) in the desired ratio are dissolved in $100 \mathrm{~mL}$ of deionized water and then placed in the flask. The total concentration of the monomers is kept constant at $1.0 \mathrm{M}$. The flask is purged with a small $\mathrm{N}_{2}$ stream for half an hour prior to being heated to $50^{\circ} \mathrm{C}$. Polymerization is then initiated by addition of $0.35 \mathrm{~mL}$ of $\mathrm{V}$ 50 ( $1 \mathrm{wt} \%$ preferentially dissolved in the deionized water) via a pipette scaled to $1 \mathrm{~mL}$ under slow stirring. In this case, instead of potassim persulfate, v-50 was used as initiator to avoid the redox side reaction between mercaptan and persulfate. Polymerization is conducted continuously for $8 \mathrm{~h}$ at $50^{\circ} \mathrm{C}$. The reaction mixture is diluted with five volumes of distilled water, and two volumes of acetone are then added with stirring to precipitate the polymers. The precipitated polymers are further washed twice with acetone and extracted with ethanol to remove all traces of water, surfactant, residual monomers, and initiator. The polymers are recovered by freeze-drying after vacuum-drying at $50^{\circ} \mathrm{C}$ for three days. For reference, PAM is prepared under identical experimental conditions and purification method mentioned above.

\subsection{Characterization}

2.5.1. Spectroscopic Measurement. IR was carried out using Shimadzu-1800S spectrometer on $\mathrm{KBr}$ pellets in the range of $4000-400 \mathrm{~cm}^{-1}$. The peak intensities are characterized as follows: $\mathrm{vs}=$ very strong, $\mathrm{s}=$ strong, $\mathrm{w}=$ weak, $\nu$ corresponds to stretching elongation, and $\delta$ corresponds to bending elongation. ${ }^{1} \mathrm{H}$ NMR spectra were measured with a Bruker AVanceII-400NMR spectrometer using $\mathrm{D}_{2} \mathrm{O}$ as a solvent at room temperature. Chemical shifts were determined by using TMS as an internal standard. The peaks multiplicities are characterized as follows: $\mathrm{s}=$ singlet, $\mathrm{d}=$ doublet, $\mathrm{t}=$ triplet, $\mathrm{q}=$ quartet, and $\mathrm{m}=$ multiplet.

2.5.2. Molecular Weights. The intrinsic viscosities $[\eta]$ of the polymers were determined by an automatic capillary viscometer (Ubbelohde type) in $0.1 \mathrm{M} \mathrm{NaCl}$ aqueous solution at room temperature. The molecular weight of each polymer sample was determined in water/methanol mixture $(3 / 7 v / v)$ containing $0.1 \mathrm{M} \mathrm{NaCl}$ by classical light scattering using a multiangle spectrometer (AMTEC model MM1) at $633 \mathrm{~nm}$. The refractive index increment $(d n / d c)$ was measured at the same wavelength on a differential refractometer. The $d n / d c$ value for PAM sample was equal to $0.16 \mathrm{~mL} \mathrm{~g}^{-1}$ [2].

2.5.3. Rheological Measurements. Polymer solutions were prepared by dissolution of a known amount of the polymer powder in water and $\mathrm{NaCl}$ solution. The apparent viscosity of samples solution at low concentrations was determined by a Brookfield DV-III rheometer equipped with different sizes of spindles (different diameter depending on solution viscosity) at $25^{\circ} \mathrm{C}$. Rheological experiments were carried out with a RS100 controlled-stress rheometer equipped with a cone plate geometry (angle $1^{\circ}$, diameter $35 \mathrm{~mm}$ ). We measured flow curves by increasing the shear stress in regular steps and waiting at each step until equilibrium was attained. The shear rate $\dot{\gamma}$ ranged from $0.1-10$ to $1000 \mathrm{~s}^{-1}$ according to the viscosity behavior of samples. To overcome the problem that a slight increase in stress caused a large jump in shear rate, we used controlled-rate mode of the rheometer which permits us to scan the whole shear rate range without large gaps. In all cases, we checked that the curves measured in controlled-stress and controlled-rate mode were well superposed. Dynamic measurements were conducted under strains and at frequencies of $0.01-100 \mathrm{~Hz}$ to lead to a linear response. All the experiments were conducted with a solvent trap to avoid any evaporation.

2.5.4. Morphology. The microstructure of polymers in both aqueous and salt solutions was observed by environment scanning electron microscope (ESEM XL 30). The sample solutions were maintained at $-3.5^{\circ} \mathrm{C}$ and the pressure was controlled below 5 Torr to keep samples solution state during the whole observation. 
2.5.5. Fluorescence. Fluorescence spectra were measured on a Lengguang 970CRT fluorescence spectrometer. All measurements were conducted at ambient temperature. The slit width of excitation and emission was kept at $5 \mathrm{~nm}$ during experiments. The excitation wavelength $(\lambda)$ was set at $339 \mathrm{~nm}$. The ration of the intensities of the third $I_{3}(385 \mathrm{~nm})$ to first $I_{1}$ $(375 \mathrm{~nm})$ vibronic peak of fluorescence spectrum of pyrene probe was used as an estimate of micropolarity of pyrene microenvironment.

\section{Result and Discussion}

3.1. Characterization of DMDCC-SDS Mixed Micelles. SDS was chosen to study the micellar behavior of DMDCC-SDS binary mixed system, because it bears the same hydrophobic long chain as DMDCC and the most common surfactant used in radical micellar copolymerization, and, most importantly, mixture of cationic (DMDCC) and anionic (SDS) surfactants has a net interaction $(\beta<0)$. In the case of binary mixed micelles composed of cationic-anionic pairs, the CMC of the surfactant mixture can be calculated as follows:

$$
\frac{1}{\mathrm{CMC}_{m}}=\frac{\alpha_{1}}{f_{1}^{m} \mathrm{CMC}_{1}}+\frac{\alpha_{2}}{f_{2}^{m} \mathrm{CMC}_{2}},
$$

where $\mathrm{CMC}_{m}$ is the critical micelle concentration of binary mixed surfactants, the subscripts 1,2 represent DMDCC and SDS, respectively. $\mathrm{CMC}_{1}, \mathrm{CMC}_{2}$ are the critical micelle concentration of single surfactant species, $\alpha_{1}$ and $\alpha_{2}$ are the bulk mole fractions of the respective surfactants in the system, and $f_{1}^{m}, f_{2}^{m}$ are the activity coefficients that can be found by using the regular solution theory,

$$
\begin{aligned}
& \ln f_{1}^{m}=\left(\alpha_{2}^{m}\right)^{2} \beta, \\
& \ln f_{2}^{m}=\left(\alpha_{1}^{m}\right)^{2} \beta,
\end{aligned}
$$

where $\beta$ is an interaction parameter, quantifying the net interaction between the surfactant species in the mixed micelle. $\alpha_{1}^{m}$ and $\alpha_{2}^{m}$ are the mole fractions of the single surfactant in mixed system which are written as follows:

$$
\begin{aligned}
\alpha_{1}^{m} & =\frac{\alpha_{1} f_{2}^{m} \mathrm{CMC}_{2}}{\alpha_{1} f_{2}^{m} \mathrm{CMC}_{2}+\alpha_{2} f_{1}^{m} \mathrm{CMC}_{1}}, \\
\alpha_{2}^{m} & =1-\alpha_{1}^{m} .
\end{aligned}
$$

Figure 1 shows the $\mathrm{CMC}_{m}$ of DMDCC-SDS binary mixed system as a function of DMDCC mole fraction in solution. We can see that the CMC values of single SDS and DMDCC in aqueous solution are $8 \mathrm{mM}, 0.82 \mathrm{mM}$, respectively. For the DMDCC-SDS mixed system, the CMC values of anionic surfactant SDS significantly decrease by adding a small amount of DMDCC without causing any precipitation. Interestingly, the $\mathrm{CMC}$ values of the binary surfactant remain almost constant with the increase in DMDCC molar fraction in aqueous solution.

The CMC of mixed surfactants strongly depends on parameter values $\beta$. Positive $\beta$ values imply a net repulsive force between the surfactant components, whereas negative $\beta$

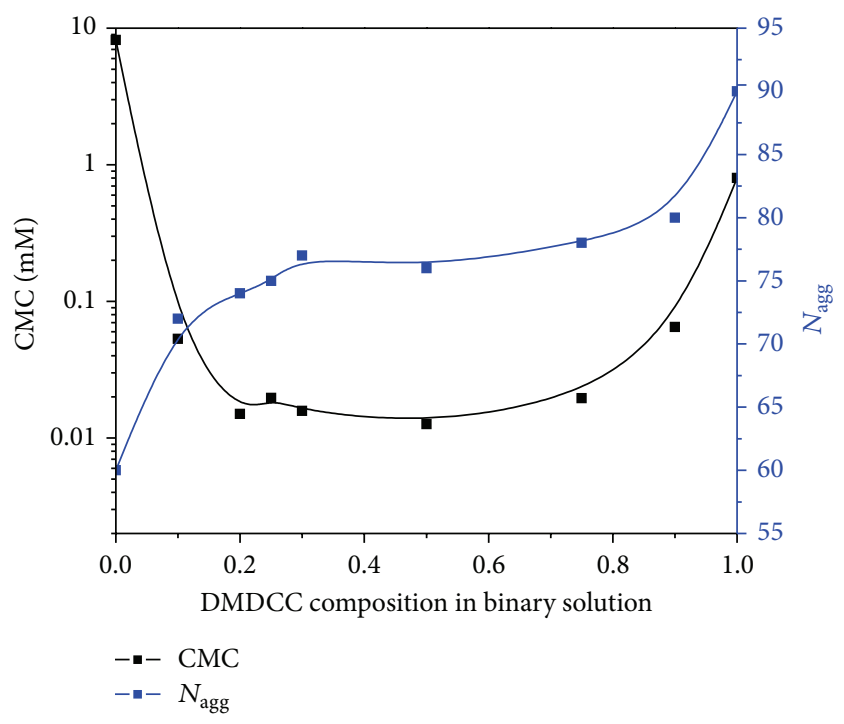

FIGURE 1: Variation of critical micelle concentration of DMDCCSDS binary mixture system as a function of DMDCC mole fraction $\left(T=50^{\circ} \mathrm{C}\right)$.

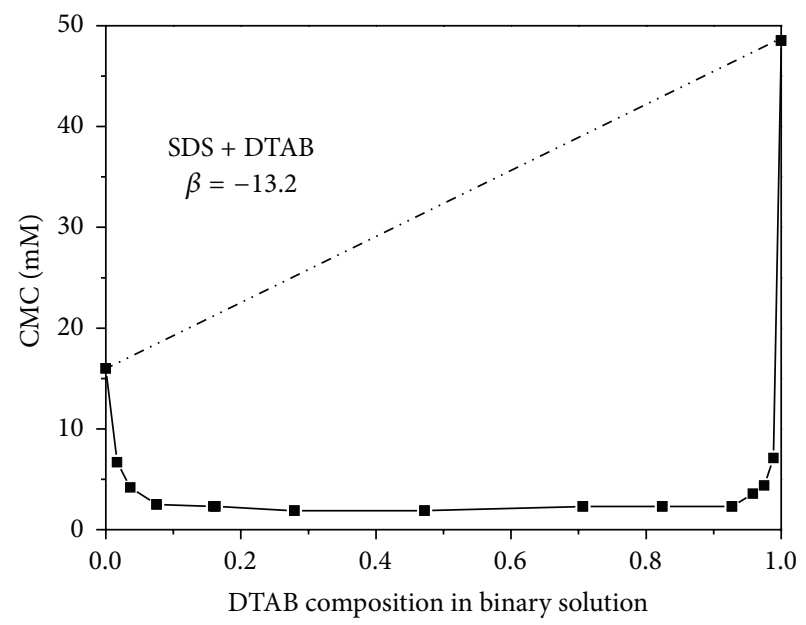

FIGURE 2: Variation of critical micelle concentration of DTAB-SDS binary mixture system as a function of DTAB mole fraction [22], reproduced from [22], with permission from John Wiley \& Sons.

values imply a net attractive force. The more negative $\beta$ values are, the stronger the net interaction between the surfactants in the mixed system will become; thus the CMC of mixed surfactants is much lower. For reference, the CMC of DTABSDS mixed system as the function of CTAB mole fraction is depicted in Figure 2 . The strong synergetic effect $(\beta=-13.2)$ indicated that there was a large net attraction between the surfactants.

The much lower CMC values of SDS are obtained by adding cationic surfactant DTAB. We note that DMDCC bears almost the same structure as DTAB. Therefore, we can draw a conclusion that the CMC behavior of DMDCC-SDS mixed system, as shown in Figure 3, has the same dynamic character as that of DTAB-SDS. However, the aggregation 


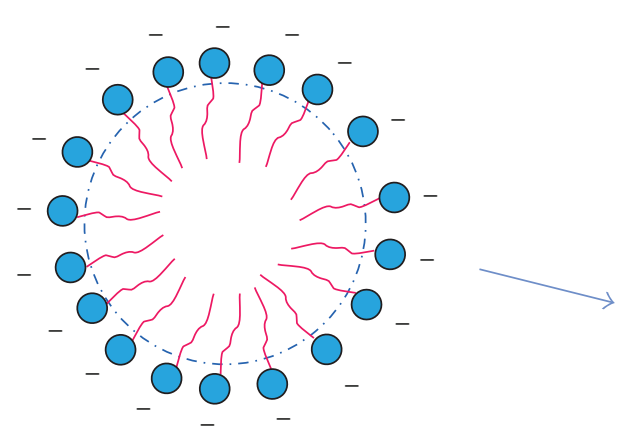

Pure SDS micelles

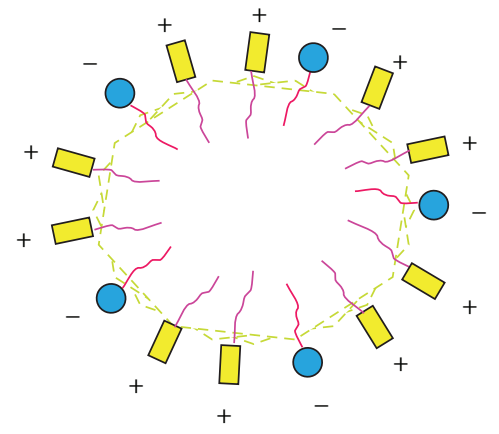

Mixed DMDCC-SDS micelles

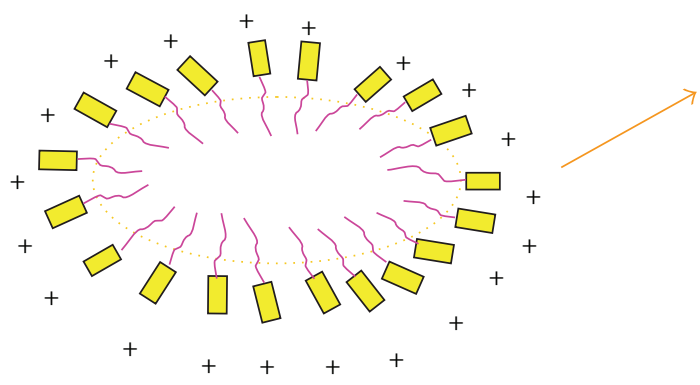

Pure DMDCC micelles

FIGURE 3: Schematic representation of the mixed DMDCC-SDS micelles compared to pure DMDCC micelles and pure SDS micelles.

morphology of DMDCC-SDS mixed system as the mole fraction of DMDCC has unique dynamic character, which is shown in Figure 1. The $N_{\mathrm{agg}}$ value of anionic surfactant SDS significantly increases by adding a small amount of DMDCC and then remains almost constant with the increase in DMDCC molar fraction in aqueous solution, which could be written as follows:

$$
N_{\mathrm{agg}}^{m}=\alpha_{1}^{m} N_{\mathrm{agg} 1}+\alpha_{2}^{m} N_{\mathrm{agg} 2},
$$

where $N_{\mathrm{agg} 1}$ and $N_{\mathrm{agg} 2}$ are the respective aggregation number values of DMDCC and SDS in aqueous solution. The length of hydrophobic microblock in hydrophobically modified polyacrylamide prepared by conventional radical micelle copolymerization is determined by

$$
N_{H}=\frac{[H] N_{\mathrm{agg}}}{[S]-\mathrm{CMC}},
$$

where $[H]$, $[S]$, and CMC are the mole concentration of the hydrophobic monomer, the mole concentration of surfactant, and critical concentration of surfactant, whereas the length of hydrophobic microblock from mixed micelle copolymerization should be rewritten as

$$
N_{H}=\frac{\left([H]-\mathrm{CMC}_{H}\right) N_{\mathrm{agg}}^{m}}{[H+S]-\mathrm{CMC}_{(H+S)}} .
$$

In the DMDCC/SDS mixed system, $\mathrm{CMC}_{H}$ is the CMC of surfmer and $\mathrm{CMC}_{H+S}$ is the critical micelle concentration of DMDCC-SDS binary mixed micelles and $N_{\text {agg }}^{m}$ which is determined by steady-state fluorescence quenching technique. Therefore, the length of microblock could be tuned reasonably by the mixed micelle technique.

3.2. Synthesis and Characterization. The copolymers poly (AM/DMDCC) with low amounts of cationic hydrophobe were obtained in water using a DMDCC-SDS binary mixed micellar polymerization (Scheme 2). The characteristic of the copolymer is depicted in Table 1. For DMDCC, IR $(\mathrm{KBr}$, $\left.v / \mathrm{cm}^{-1}\right): 3432$ (s, $\left.v(\mathrm{C}=\mathrm{O})\right), 2919$ (vs, $\left.v_{\text {as }}(\mathrm{C}-\mathrm{H})\right), 2844$ (s, $\left.v_{\mathrm{s}}(\mathrm{C}-\mathrm{H})\right), 1633(\mathrm{w}, v(\mathrm{C}=\mathrm{C})), 1299(\mathrm{~s}, \nu(\mathrm{C}-\mathrm{N})), 1167$ (vs, $\nu(\mathrm{C}-$ C)) $1028(\mathrm{w}, \nu(\mathrm{C}-\mathrm{O}))$. For copolymer 0.75DM12.8, IR ( $\mathrm{KBr}$, $\left.v / \mathrm{cm}^{-1}\right): 3450$ (vs, $\left.v_{\mathrm{s}}\left(\mathrm{NH}_{2}\right), v(\mathrm{C}=\mathrm{O})\right), 2925(\mathrm{w}, \nu(\mathrm{C}-\mathrm{H})), 1025$ $(\mathrm{w}, \nu(\mathrm{C}-\mathrm{O})), 628(\mathrm{~s}, \delta(\mathrm{C}-\mathrm{H})) .{ }^{1} \mathrm{H}$ NMR $\left(400 \mathrm{MHz}, \mathrm{D}_{2} \mathrm{O}\right.$, $\delta / \mathrm{ppm}): 1.11\left(\mathrm{q}, 2 \mathrm{H}_{\mathrm{g}} ; \mathrm{CH}_{2}\right), 1.58\left(\mathrm{~d}, 2 \mathrm{H}_{\mathrm{a}} ; \mathrm{CH}_{2}\right), 2.16\left(\mathrm{~d}, 1 \mathrm{H}_{\mathrm{b}}\right.$, $\mathrm{CH}), 2.36\left(\mathrm{t}, 3 \mathrm{H}_{\mathrm{c}}, \mathrm{CH}_{3}\right), 2.48\left(\mathrm{~s}, 3 \mathrm{H}_{\mathrm{f}}, \mathrm{CH}_{3}\right), 3.59\left(\mathrm{~m}, 2 \mathrm{H}_{\mathrm{e}}\right.$, $\left.\mathrm{CH}_{2}\right), 3.72\left(\mathrm{q}, 2 \mathrm{H}_{\mathrm{d}}, \mathrm{CH}_{2}\right.$ ) (Figures 4 and 5).

\subsection{Rheological Behavior}

3.3.1. Viscometric Behavior in Dilute Solution. The effect of the hydrophobic microblock length, that is, $N_{H}$ value, and the concentration of hydrophobic monomer on intra- and intermolecular interaction is depicted in Figure 6. Apparent viscosity is plotted as a function of polymer concentration for three copolymers (0.5DM4.5, 0.5DM9.3, and 0.75DM9.3) and for the corresponding polyacrylamide.

All the samples are well measured at a constant shear rate of $7.34 \mathrm{~s}^{-1}$. In extremely dilute solution regime 
TABLE 1: Structural parameters of the copolymers.

\begin{tabular}{|c|c|c|c|c|c|c|c|c|c|}
\hline \multirow{2}{*}{ Sample ${ }^{a}$} & \multicolumn{3}{|c|}{ Polymer composition mol \% } & \multirow{2}{*}{$N_{H}^{\mathrm{b}}$} & \multicolumn{5}{|c|}{ Polymer characterization } \\
\hline & $\mathrm{AM}$ & DMDCC & SDS & & $S^{c}$ & $\begin{array}{c}10^{-6} \times M_{w} \\
\mathrm{~g} \mathrm{~mol}^{-1}\end{array}$ & $\begin{array}{c}{[\eta]} \\
\mathrm{mLg}^{-1}\end{array}$ & $\begin{array}{l}{[\eta]_{\text {call }^{d}}} \\
\mathrm{~mL} \mathrm{~g}^{-1}\end{array}$ & Solubility in water ${ }^{\mathrm{e}}$ \\
\hline PAM & 100 & 0 & 0 & 0 & 0 & 3.50 & 610 & 755 & ++ \\
\hline $0.5 \mathrm{DM} 4.5$ & \multirow{4}{*}{99.5} & \multirow{4}{*}{0.5} & 1.75 & 4.5 & 25 & 3.15 & 586 & 697 & ++ \\
\hline $0.5 \mathrm{DM} 9.3$ & & & 0.8 & 9.3 & 12.4 & 3.29 & 540 & 720 & + \\
\hline $0.5 \mathrm{DM} 12.7$ & & & 0.5 & 12.7 & 9 & 3.25 & 520 & 714 & + \\
\hline $0.5 \mathrm{DM} 92$ & & & 0 & 92 & 0.6 & 1.64 & 299 & 428 & \pm \\
\hline $0.75 \mathrm{DM} 4.6$ & \multirow{4}{*}{99.25} & \multirow{4}{*}{0.75} & 5.5 & 4.6 & 25 & 2.22 & 335 & 536 & ++ \\
\hline $0.75 \mathrm{DM} 9.3$ & & & 2.1 & 9.3 & 17 & 3.06 & 470 & 683 & + \\
\hline $0.75 \mathrm{DM} 12.8$ & & & 1.35 & 12.8 & 13 & 3.35 & 418 & 730 & + \\
\hline $0.75 \mathrm{DM} 92$ & & & 0 & 92 & 0 & 1 & 1 & 1 & - \\
\hline
\end{tabular}

${ }^{\mathrm{a}}$ The first number indicated the mole frication of hydrophobic monomer, and the final number indicated the rounded value of $N_{H}$ (e.g., $0.5 \mathrm{DM} 5$ stands for a polymer that contains $0.5 \mathrm{~mol} \%$ of DMDCC with $\left.N_{H} \approx 4.5\right)$. ${ }^{\mathrm{b}}$ Number of hydrophobes per micelle $\approx$ hydrophobic block length. ${ }^{\mathrm{c}}$ Number of hydrophobic blocks per chain (i.e., stickers) ${ }^{30}$ calculated using the relationship $S=\left(M_{W} / 2 m\right)[H] / N_{H}$, where $m$ is the molecular weight of the monomer unit (acrylamide, $m$ $=71)$ and $M_{W} / 2 m$ corresponds to the number average degree of polymerization, $N$. ${ }^{\mathrm{d}}$ Intrinsic viscosity calculated using relationship $[\eta]=9.33 \times 10^{-3} M_{W}{ }^{0.75}$ established for PAM ${ }^{31} .{ }^{e}++$, easily soluble; +, soluble; \pm , cloudy solution; -, difficult to dissolve.

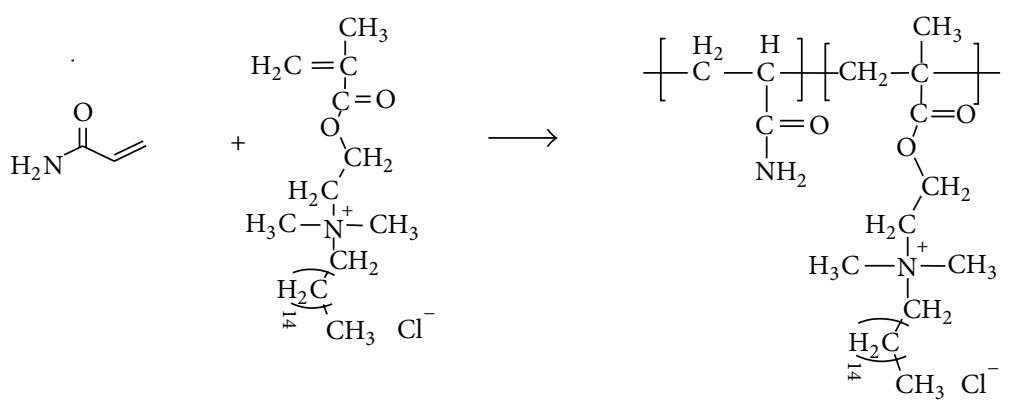

SCHEME 2: Copolymerization of AM and DMDCC.

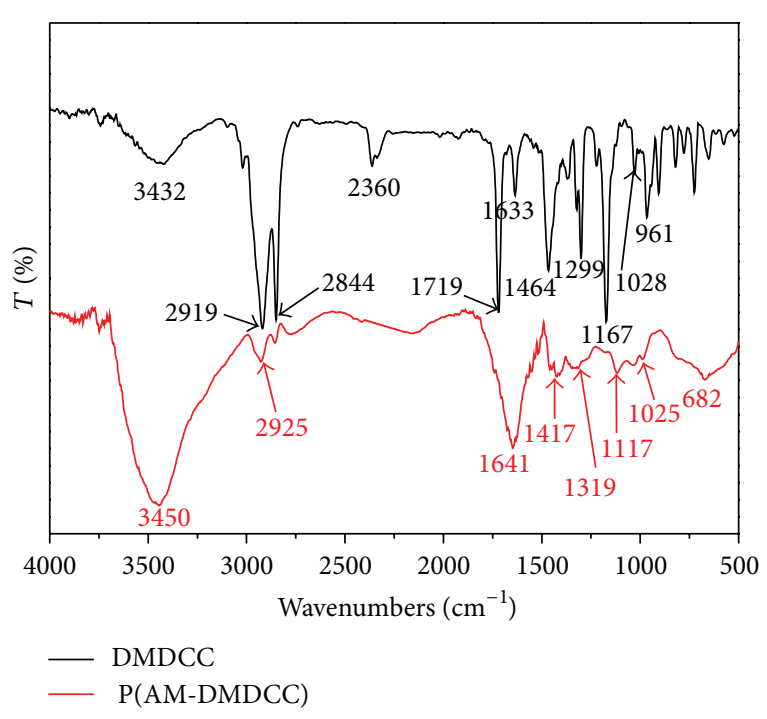

FIGURE 4: IR spectra of the DMDCC and copolymer 0.75DM12.8.
$\left(c_{P}<0.05 \mathrm{~g} \mathrm{dL}^{-1}\right)$, the apparent viscosity of copolymers is lower than that of PAM due to intramolecular association which leads to the coil of chain. This effect is enhanced by increasing either the $N_{H}$ or the hydrophobe concentration. And it is well explained that the $[\eta]$ of all the poly(AM/DMDCC) is reduced compared to that of PAM and inverse to the $N_{H}$ value. Because the $[\eta]$ of the polymer was determined by automatic capillary viscometer at low polymer concentration, intermolecular interaction was gradually enhanced with the increase in polymer concentration. Above a critical concentration, that is, CAC, an upward curvature in the apparent viscosity can be observed. The apparent viscosity increases more markedly and shows up at lower concentration with increasing the hydrophobic length at the same hydrophobe concentration. The CAC of 0.5DM4.5 and $0.5 \mathrm{DM} 9.3$ is $0.15 \mathrm{~g} \mathrm{dL}^{-1}$ and $0.13 \mathrm{~g} \mathrm{dL}^{-1}$, respectively. On the other hand, 0.75DM9.3 and 0.5DM9.3 have the same hydrophobic microblock length; the increase in hydrophobe also enhances thickening efficiency. This behavior can be 


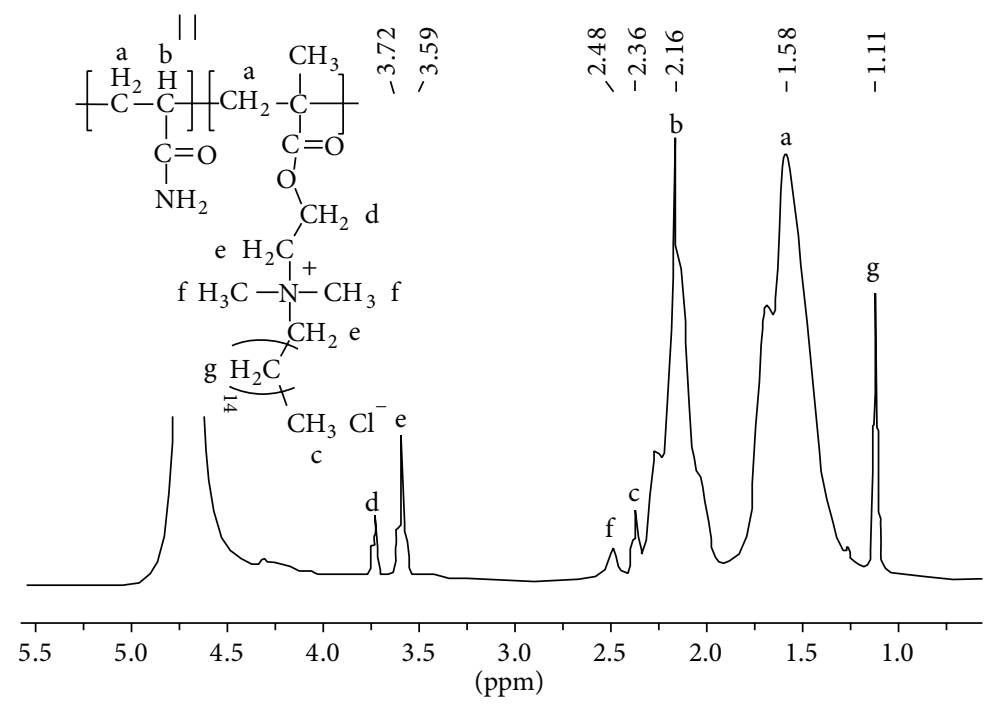

Figure 5: ${ }^{1}$ HNMR spectra of copolymer 0.75DM12.8.

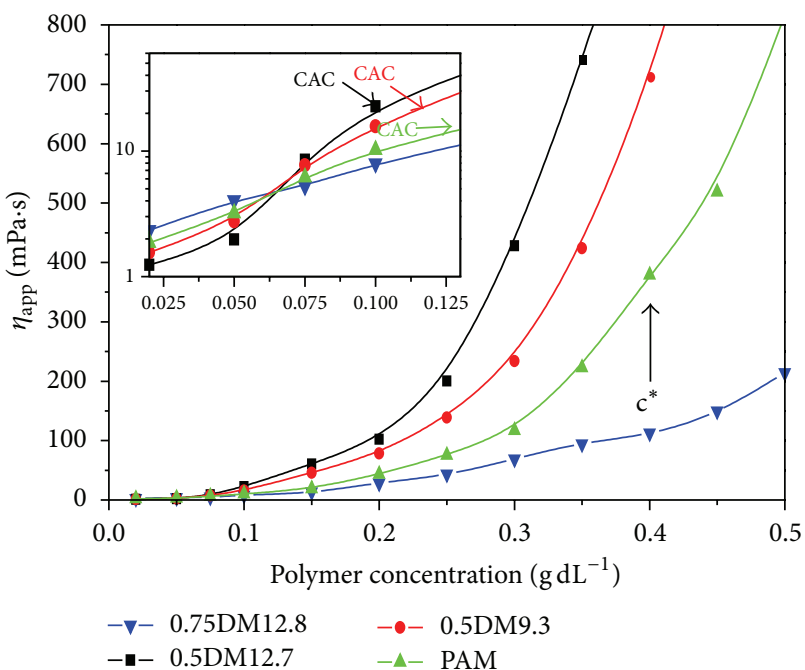

FIGURE 6: Apparent viscosity as a function of polymer concentration for copolymers 0.75DM12.8, 0.5DM12.7, and 0.5DM9.3 samples and for a PAM sample $\left(T=25^{\circ} \mathrm{C}\right.$, the inlet of apparent viscosity is partially enlarged for purpose of smart understanding).

explained by the increase in sticker reported in Table 1, which makes the intermolecular interactions much stronger, and the CAC of $0.75 \mathrm{DM} 9.3$ is $0.11 \mathrm{~g} \mathrm{dL}^{-1}$. The upturn of apparent viscosity in case of the PAM is due to the onset of chain overlap, that is, $c^{*}$, and related to the chain size. The viscosity upturn observed for poly(AM/DMDCC) cannot be attributed to a chain overlap since poly(AM/DMDCC) had the similar range of molecular weight. Moreover the chain overlap behavior was long-term effect which occurred at high polymer concentration. Therefore, the remarkable viscosity onset is a result of the formation of hydrophobic microdomain caused by hydrophobic intermolecular interaction. The hydrophobic microblock length directly influences the thickening efficiency. However, when the $N_{H}$ goes beyond a certain value, negative impact, such as low rate polymer conversion and the poor solubility, would occur, which accorded with the general law of the water-soluble associating polymer prepared by conventional radical micelle copolymerization $[4,16,30]$.

3.3.2. Steady Flow Experiments. Apparent viscosity as a function of shear rate is conducted for three copolymers modified by $0.5 \mathrm{~mol} \%$ DMDCC and for PAM. The viscosity/shear rate curves of the copolymers and PAM at concentration of $1.0 \mathrm{~g} \mathrm{dL}^{-1}$ and $2.0 \mathrm{~g} \mathrm{dL}^{-1}$ are shown in Figures 7 and 8 . It is interesting to figure out that the rheological properties of the copolymers are strongly dependent on $N_{H}$ and polymer concentration.

PAM exhibits the classic rheological behavior, that is, a slight shear thinning effect after Newtonian plateau. All the copolymers present the same type of behavior. For poly(AM/DMDCC), at $1.0 \mathrm{~g} \mathrm{dL}^{-1}$ concentration, a shear thickening domain reaches first followed by shear thinning. The shear thickening is interpreted in terms of the balance between intra- and intermolecular associations. At a certain shear rate, the shear forces are strong enough to extend the polymer coils and disrupt the intramolecular hydrophobic interactions. The intramolecular hydrophobic microdomain is released and favors forming intermolecular hydrophobic interactions, resulting in an increase in viscosity. Further increasing shear rate, these interactions are gradually broken and shear thinning behavior is observed. This behavior was general agreement with the nonionic hydrophobically modified polyacrylamides [31].

To determine the role of the $N_{H}$ in the apparent viscosity, for all polymers, the values of viscosity $\left(\eta_{0}\right)$ which corresponded to the shear rate $\left(\dot{\gamma}^{*}\right)$ and shear stress $\left(\sigma^{*}\right)$ on the onset of Newtonian plateau were reported in Table 2. Apparent viscosity increases with the increasing of shear rate; 
TABLE 2: Rheological values of $\sigma^{*}, \dot{\gamma}^{*}$, and $\eta_{0}$ for poly(AM/DMDCC) and PAM at concentration of $1.0 \mathrm{~g} \mathrm{dL}^{-1}$ and $2.0 \mathrm{~g} \mathrm{dL}^{-1}$.

\begin{tabular}{|c|c|c|c|c|c|c|}
\hline \multirow{2}{*}{ Polymer } & \multicolumn{3}{|c|}{$c_{p}=1.0 \mathrm{~g} \mathrm{dL}^{-1}$} & \multicolumn{3}{|c|}{$c_{p}=2.0 \mathrm{~g} \mathrm{dL}^{-1}$} \\
\hline & $\sigma^{*}(\mathrm{~Pa})$ & $\dot{\gamma}^{*}\left(\mathrm{~s}^{-1}\right)$ & $\eta_{0}(\mathrm{mPa} \cdot \mathrm{s})$ & $\sigma^{*}(\mathrm{~Pa})$ & $\dot{\gamma}^{*}\left(\mathrm{~s}^{-1}\right)$ & $\eta_{0}(\mathrm{mPa} \cdot \mathrm{s})$ \\
\hline PAM & 0.93 & 10.3 & 90 & 3.6 & 10 & 357 \\
\hline $0.5 \mathrm{DM} 4.5$ & 1.09 & 1.66 & 658 & 8.2 & 0.56 & 14650 \\
\hline $0.5 \mathrm{DM} 9.3$ & 1.55 & 1.23 & 1264 & 9.78 & 0.36 & 27160 \\
\hline $0.5 \mathrm{DM} 12.7$ & 2.07 & 0.76 & 2723 & 13.15 & 0.25 & 62600 \\
\hline
\end{tabular}

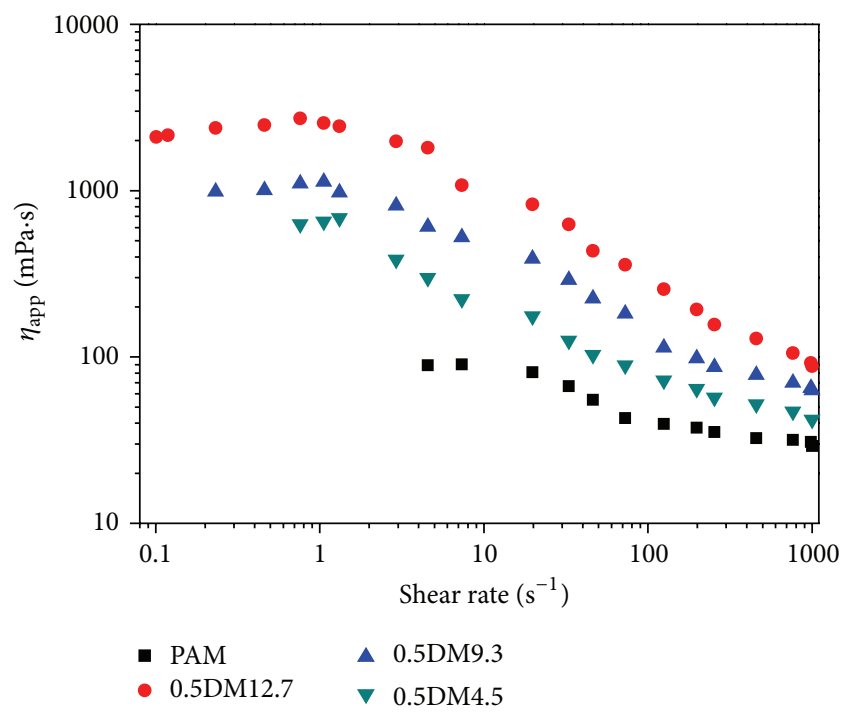

FIGURE 7: Variation of apparent viscosity as a function of shear rate for PAM, 0.5DM12.7, and 0.5DM4.5 $\left(c_{p}=1.0 \mathrm{~g} \mathrm{dL}^{-1}, T=25^{\circ} \mathrm{C}\right)$.

at such shear rate $\left(\dot{\gamma}^{*}\right)$, this plateau is attainable as shown in Figures 7 and 8, and beyond which the fluid presents a shear thinning behavior; the values of the stress threshold $\sigma^{*}=\dot{\gamma}^{*} \eta_{0}$.

In case of PAM, a higher $\dot{\gamma}^{*}$ is presented compared to that of copolymers. With the increase in shear rate, shear force is strong enough to disrupt the gauss coils structured by the macromolecular chains overlap and entanglement, and the original chain orientation is released to form orderly orientation, resulting in a decrease in viscosity. As for copolymers, the length of hydrophobic microblock 4.5 (0.5DM4.5) is enough to induce an increase in the solution viscosity. Lengthening of hydrophobic microblock enhanced the degree of association facilitating shear thickening behavior. At a certain shear rate, the shear forces are strong enough to extend the copolymer coils and disrupt the intramolecular hydrophobic interactions, resulting in the decrease of viscosity.

The stress threshold values $\sigma^{*}$ show that the longer the $N_{H}$, the higher the $\sigma^{*}$. This indicates that more stress force is required to disrupt the molecular interactions with increase in the length of hydrophobic microblock, which results in a stronger transitory three-dimensional network. At the identical polymer concentration, the increase in $N_{H}$ is accompanied by a decrease of $\dot{\gamma}^{*}$, shortening the Newtonian

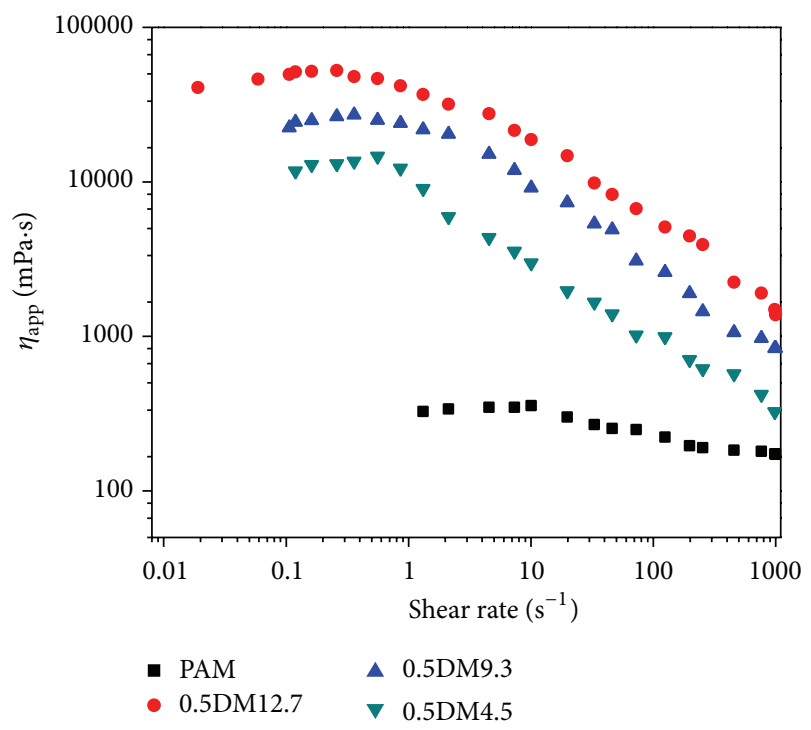

FIGURE 8: Variation of apparent viscosity as a function of shear rate for PAM, 0.5DM12.7, and 0.5DM4.5 $\left(c_{p}=2.0 \mathrm{~g} \mathrm{dL}^{-1}, T=25^{\circ} \mathrm{C}\right)$.

zone. The solution became more sensitive to shear rate. For example, at a concentration of $1.0 \mathrm{~g} \mathrm{dL}^{-1}$, the shear rate $\dot{\gamma}^{*}$ of copolymers $0.5 \mathrm{DM} 12.7$ and $0.5 \mathrm{DM} 9.3$ is $0.76 \mathrm{~s}^{-1}$ and $1.23 \mathrm{~s}^{-1}$, respectively, compared to $1.66 \mathrm{~s}^{-1}$ of copolymer $0.5 \mathrm{DM} 4.5$. Higher viscosity at low shear rate indicates a more pronounced shear thickening domain and a longer shear thinning plateau. At higher shear rate, the copolymer viscosity trends towards that of PAM, but even at $1000 \mathrm{~s}^{-1}$, the value is mainly greater indicating that intermolecular interaction also existed.

We should figure out that even at a higher concentration $\left(2.0 \mathrm{~g} \mathrm{dL}^{-1}\right)$, shear thickening domain is also observed, and both copolymers show classical shear thickening subsequent shear thinning behavior within the range of shear rate. This owning to the fact that beside molecular weight and polymer concentration the nature of hydrophobic monomer also influences shear thickening efficiency. The apparent viscosity/shear rate curves at the characteristic values at the Newtonian plateau are also given in Table 2. We also observe that increasing the concentration shortens the shear thickening plateau, while the magnitude of the $\eta_{0}$ increases with increasing polymer concentration. The values reported in Table 2 confirmed that, for cationic hydrophobic associative polyacrylamides, concentration, especially, $N_{H}$, had great effects on steady flow rheological properties. 
3.3.3. Linear Viscoelasticity. Figures 9 and 10 present illustration of the oscillatory response for poly(AM/DMDCC) and PAM at $1.0 \mathrm{~g} \mathrm{dL}^{-1}$ in aqueous solution. The variations of the storage $\left(G^{\prime}\right)$ and loss $\left(G^{\prime \prime}\right)$ modulus are curved versus frequency $(f)$. The slopes of $G^{\prime}$ and $G^{\prime \prime}$ in the terminal zone for polyacrylamide are 2.2 and 0.98 . Note that the slopes of $G^{\prime}$ and $G^{\prime \prime}$ will not keep constant, which are dependent on the PAM concentration [4].

However, for copolymers modified by hydrophobic groups, at low frequencies, the behavior of the shear modulus is Maxwellian, indicating the variations of $G^{\prime}(f)$ and $G^{\prime \prime}(f)$ that scaled as $f^{2}$ and $f$, respectively. The curves obtained for the different series of copolymers have a profile similar to that for solutions whose viscoelastic properties are governed by chain entanglements. These solutions are characterized at low frequency by a slope of $G^{\prime}$ and $G^{\prime \prime}$, where the values of $G^{\prime}$ were below that of $G^{\prime \prime}$. Above the frequency at which curves $G^{\prime}(f)$ and $G^{\prime \prime}(f)$ cross each other $\left(G_{c}\right)$, the shape of these curves deviates from Maxwellian behavior, indicating the presence of faster relaxation processes, which superimpose each other on the main slow relaxation process. This behavior can be interpreted in terms of the copolymers polydispersity and imperfections in the macromolecular microstructure, which should inevitably broaden the relaxation spectra. The dynamics of the system are scaled by the slope of $G^{\prime}$ and $G^{\prime \prime}$ in the terminal zone, $G_{c}$, and characteristic time $t_{c}(1 / f)$ at a frequency corresponding to the crossing point $G_{c}$. According to the sticky reputation theory of Leibler et al. [32], the value of $t_{c}$ indicated the disentanglement time of polymer chain limited outside the tube. The values obtained are reported in Table 3.

The presence of the hydrophobic group into macromolecular chain increased both moduli and the terminal zone is shifted toward low frequencies due to the transitional network junctions by hydrophobic associations. 0.5 DM4.5 exhibits a slight increase in the modulus at $\mathrm{Hz}$ compared to that of PAM, while copolymer 0.5 DM9.3 shows a more marked enhancement with $G_{c}=2.27 \mathrm{~Pa}$ at $1.17 \mathrm{~Hz}$. Increasing the microblock length of the hydrophobe or its hydrophobic molar proportion in the polymer is accompanied by the increase in the modulus and the slower relaxation time. Both the storage $\left(G^{\prime}\right)$ and loss $\left(G^{\prime \prime}\right)$ modulus reflected the strength of the intermolecular interactions. At the identical hydrophobic proportion, $G_{c}\left(G^{\prime}=G^{\prime \prime}\right)$ of $0.75 \mathrm{DM} 9.3$ is 2.78 Pa compared to $1.79 \mathrm{~Pa}$ of 0.75DM4.6. Also, in consideration of the similar $N_{H}$ the value of $G_{c}$ was, respectively, 2.45 Pa and 3.56 Pa for 0.5DM12.7 and 0.75DM12.8. Note that the storage $\left(G^{\prime}\right)$ and loss $\left(G^{\prime \prime}\right)$ modulus exhibit uncoordinated variation over the frequency considered. $G^{\prime}$ becomes larger than $G^{\prime \prime}$ after the cross-point, indicating the domination of elastic part.

On the other hand, time $t_{c}$ also increased significantly with the strength of the intermolecular associations, that is, with the lifetime of the hydrophobic groups in interaction form. For copolymers 0.5DM4.5 and 0.75DM4.6, the relaxation time is, respectively, 32 and 82 times the values for PAM. We also find the longer $N_{H}$ with less hydrophobic proportion had the equivalent $t_{c}$ to that shorter $N_{H}$ with

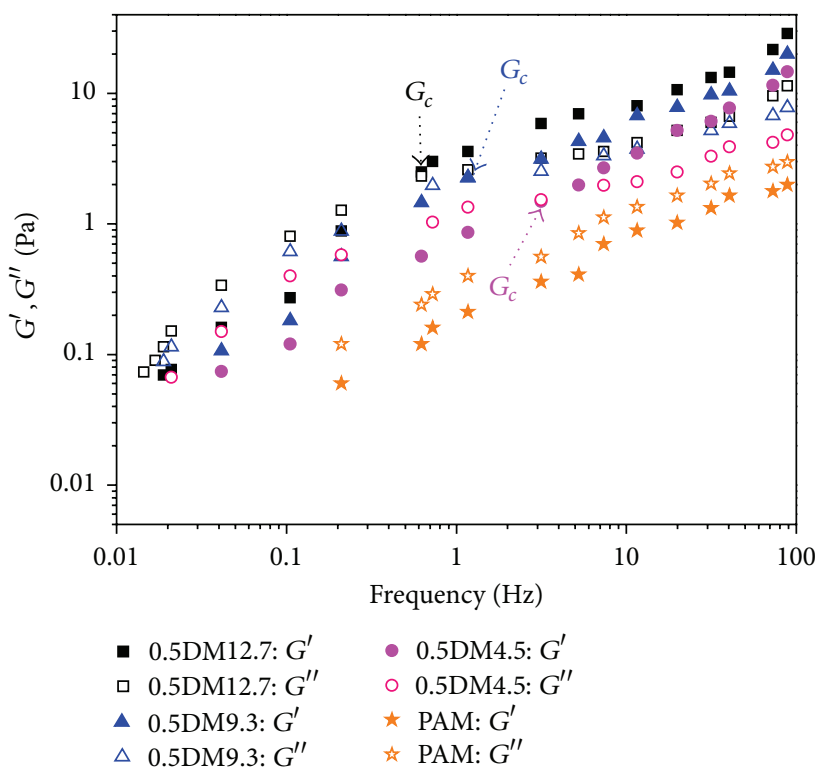

FIGURE 9: Storage $\left(G^{\prime}\right)$ and loss $\left(G^{\prime \prime}\right)$ modulus as a function of frequency for copolymers 0.5DM12.7, 0.5DM9.3, and 0.5DM4.5 and for PAM $\left(c_{p}=1.0 \mathrm{~g} \mathrm{dL}^{-1}, T=25^{\circ} \mathrm{C}\right)$.

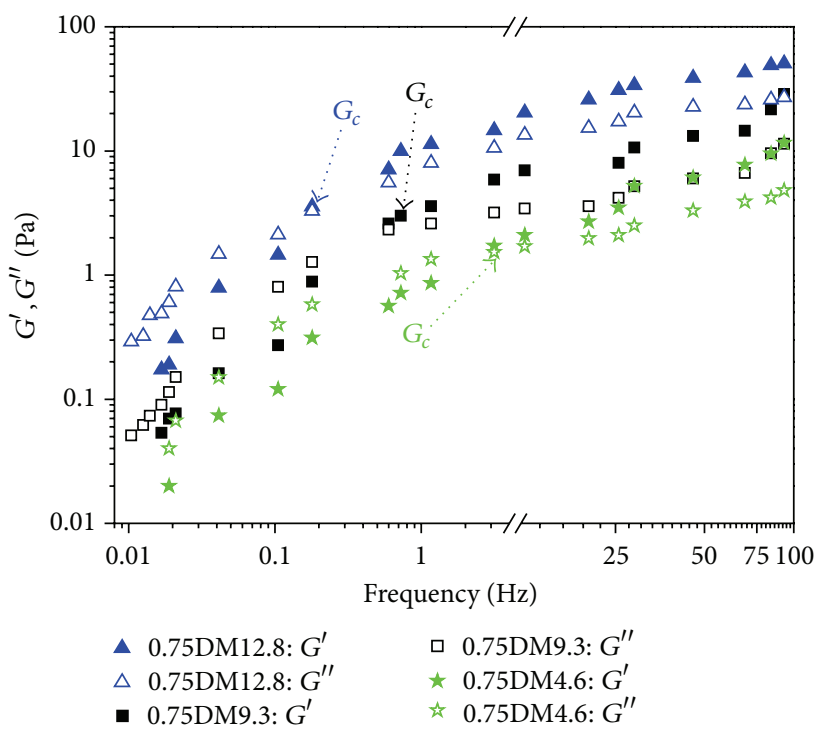

Figure 10: Storage $\left(G^{\prime}\right)$ and loss $\left(G^{\prime \prime}\right)$ modulus as a function of frequency for copolymers 0.75DM12.8, 0.75DM9.3, and 0.75DM4.6 and for PAM $\left(c_{p}=2.0 \mathrm{~g} \mathrm{dL}^{-1}, T=25^{\circ} \mathrm{C}\right)$.

more hydrophobic proportion. The relaxation time is $0.82 \mathrm{~s}$ for 0.75DM4.6 to $0.85 \mathrm{~s}$ for 0.5DM9.3. Moreover, at the same hydrophobic proportion, the increase in $t_{c}$ was even more pronounced when $N_{H}$ increases. The second relaxation time is not observed and it will probably be located at higher frequencies which is experimentally inaccessible since the response is no longer linear. 
TABLE 3: Values of the slopes of the storage modulus $G^{\prime}$ and the loss modulus $G^{\prime \prime}$ in the terminal zone and values of the crossing time $t_{c}$ for the polymers.

\begin{tabular}{lcccc}
\hline Polymer & $G^{\prime}(\mathrm{Pa})$ & Slope & $G_{c}^{\prime \prime}(\mathrm{Pa})$ & \multicolumn{1}{c}{$t_{c}(\mathrm{~s})$} \\
\hline PAM & 2.2 & 0.98 & 1 & $<0.01$ \\
$0.5 \mathrm{DM} 4.5$ & 1.6 & 1.17 & 2.27 & 0.32 \\
$0.5 \mathrm{DM} 9.3$ & 1.35 & 1.05 & 2.45 & 0.86 \\
$0.5 \mathrm{DM} 12.7$ & 1.27 & 0.86 & 1.79 & 2.85 \\
$0.75 \mathrm{DM} 4.6$ & 1.46 & 1.15 & 2.78 & 0.82 \\
$0.75 \mathrm{DM} 9.3$ & 1.25 & 0.93 & 3.56 & 6.92 \\
$0.75 \mathrm{DM} 12.8$ & 1.32 & 0.96 & & \\
\hline
\end{tabular}

\subsection{Additives Influence}

3.4.1. Influence of Salt. The apparent viscosity as a function of $\mathrm{NaCl}$ concentration for $0.75 \mathrm{DM} 12.8$ and PAM at polymer concentration of $0.15 \mathrm{~g} \mathrm{dL}^{-1}$ and $0.75 \mathrm{~g} \mathrm{dL}^{-1}$ was represented in Figure 11. The values of the curves are expressed in terms of a relative viscosity, that is, the ratio of the viscosity in the presence of salt to the viscosity in salt-free solution.

The behavior of PAM exhibits almost the same trend at two different concentrations in the whole salt concentration range. At low salt concentration, the viscosity decreases smoothly upon the increase in salt content, and above the certain salt concentration, relative constant apparent viscosity is exhibited. For PAM, increasing the concentration slightly shifts the constant apparent viscosity towards the lower salt concentration. At the highest salt level, the viscosity of PAM at $0.75 \mathrm{~g} \mathrm{dL}^{-1}$ is about $85 \%$ of its initial value in pure water. This can be explained that the polarity of solvent is changed in the presence of salt, resulting in the oppression of macromolecular chain accompanied by slight decrease of the apparent viscosity. However, for copolymer $0.75 \mathrm{DM} 12.8$ in salt solution, the variation of the viscosity depends significantly on the polymer concentration. For copolymer $0.75 \mathrm{DM} 12.8$ dilute solution $\left(0.15 \mathrm{~g} \mathrm{dL}^{-1}\right)$, slightly higher than critical concentration $\left(0.11 \mathrm{~g} \mathrm{dL}^{-1}\right)$, apparent viscosity increases with increasing of $\mathrm{NaCl}$ concentration. At $\mathrm{NaCl}$ concentration of $0.80 \mathrm{~g} \mathrm{dL}^{-1}$, apparent viscosity reaches the maximum value (3 times that value in salt free solution), subsequently decreases steeply, and it finally becomes close to that of PAM. Solution polarity induced by the presence of $\mathrm{NaCl}$ enhances intermolecular interaction between hydrophobic groups of the chains and the good saltthickening behavior is observed. On the other hand, with further increasing the salt concentration, the hydrophobic microstructures turn compact and isolated macromolecules resulting in the disappearance of intermolecular interaction and the decrease of the apparent viscosity. This behavior supports the conclusion that, below the chain overlap regime, the thickening efficiency arises from the intermolecular interaction between the hydrophobic segments of the chains. Once the transient network is disrupted into small clusters, the viscosity is significantly lowered. Conversely, over the chain overlap regime $\left(0.75 \mathrm{~g} \mathrm{dL}^{-1}\right)$, apparent viscosity of copolymer $0.75 \mathrm{DM} 12.7$ almost keeps constant after attaining the

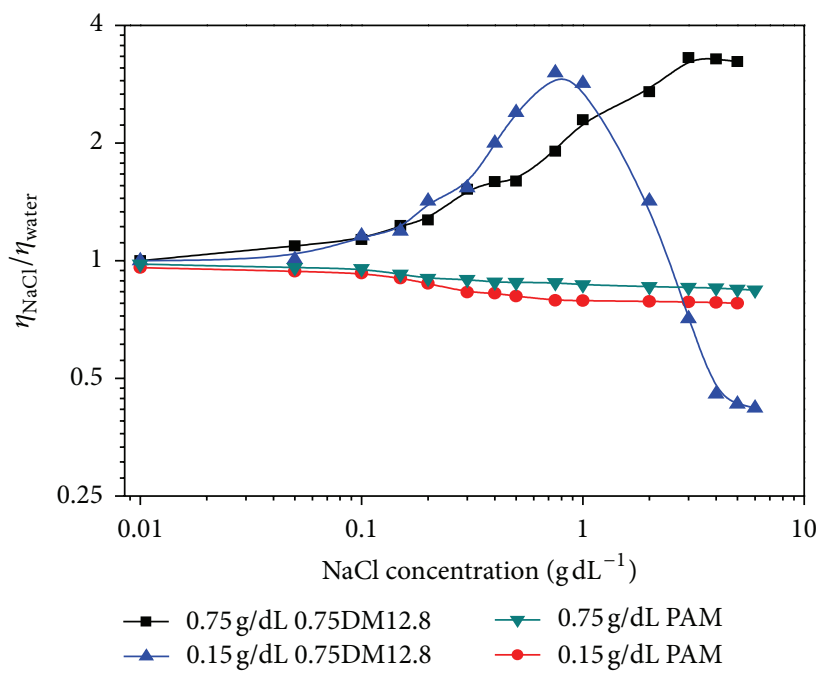

FIGURE 11: Relative variation of the viscosity with $\mathrm{NaCl}$ concentration for copolymer $0.75 \mathrm{DM} 12.8$ and PAM at two different concentrations (shear rate $7.34 \mathrm{~s}^{-1}, T=25^{\circ} \mathrm{C}$ ).

maximum value (3.5 times that value in salt-free solution). This can be ascribed to the high degree of chain overlap and even entanglement at this concentration prevents the network from disruption. For copolymer 0.75DM12.7, when the salt concentration is above $10 \mathrm{~g} \mathrm{dL}^{-1}$, either clouding or separation is observed.

3.4.2. Influence of Surfactant. Figure 12 depicts the dependence of the variation viscosity for copolymers $0.75 \mathrm{DM} 12.7$ and $0.75 \mathrm{DM} 9.3$ on the SDS concentration. SDS and hydrophobic monomer DMDCC bear the opposite charges; thus, interaction between copolymer chain and SDS occurs. Surfactant molecules first absorb at the hydrophobic units at low surfactant concentrations to form hydrophobic clusters. With continuously increasing concentration of SDS, the microdomain is reinforced by mixed micelles formed by hydrophobic groups and surfactant expanding the clusters size, resulting in the increase in viscosity. Near the CMC of SDS, significantly cooperative interaction between the copolymer chain and surfactant molecule is constructed due to mixed micelles serving as junction points for transitional 


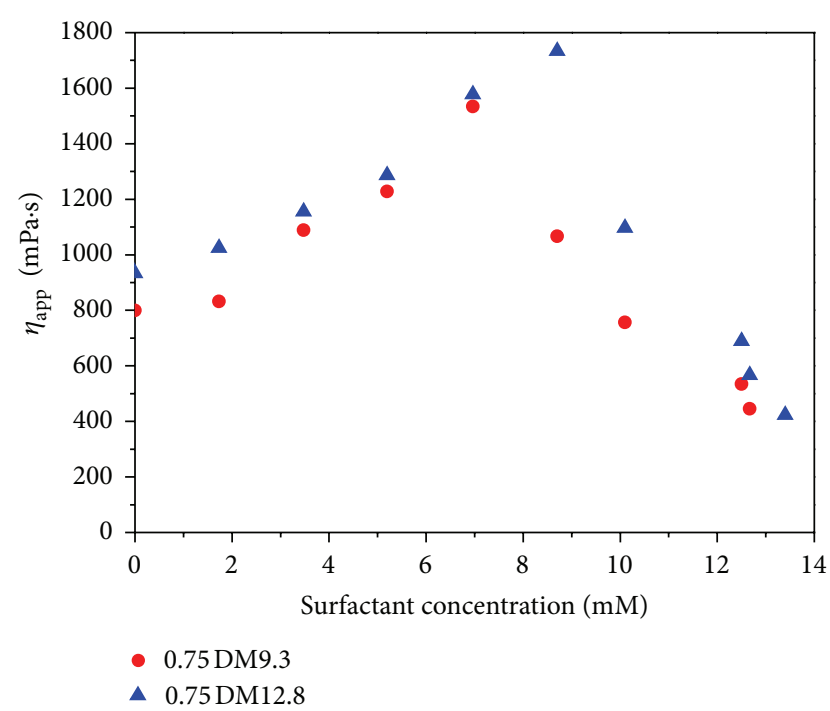

FIGURE 12: Variation of apparent viscosity of as a function of SDS concentration for copolymers $0.75 \mathrm{DM} 12.8$ and $0.75 \mathrm{DM} 9.3$ at $0.5 \mathrm{~g} \mathrm{dL}^{-1}$ (shear rate $7.34 \mathrm{~s}^{-1}, T=25^{\circ} \mathrm{C}$ ).

network. And the maximum value of viscosity for copolymer $0.75 \mathrm{DM} 12.8$ is observed (2 times that of value in SDS-free solution). Beyond the CMC of SDS, hydrophobic segments of copolymer chains are dissolved into micelles formed by much numbers of surfactant molecules, disrupting junction points, resulting in the decrease of viscosity.

It is noteworthy that the increasing of $N_{H}$ shifts the maximum viscosity toward the higher SDS concentration and more remarkable viscosity is observed. For copolymer $0.75 \mathrm{DM} 12.8$ the maximum viscosity, $1750 \mathrm{mPa} \cdot \mathrm{s}$, is exhibited at the SDS concentration of $8.5 \mathrm{mM}$ compared to $1530 \mathrm{mPa} \cdot \mathrm{s}$ for 0.75DM9.3 at the SDS concentration of $6.7 \mathrm{mM}$. Cooperatively, as the SDS concentration is raised above CMC, a large viscosity for $0.75 \mathrm{DM} 12.8$ is still observed. Two points can be used to interpret that the viscosity for 0.75DM12.8 in the presence of surfactant is much higher than that of value for 0.75DM9.3, that is, hydrophobic association and ionic attraction. Hydrophobic associations reduce the contacts of the hydrophobic segments of polymer chain with water leading to lowering the free energy of the system [3]. It is discussed above that the longer the hydrophobic microblock, the stronger the intermolecular association, and thus, less free energy of the system. Ionic attraction between SDS molecules and copolymer chains is obviously enhanced with prolonging the length of cationic hydrophobic groups per chain. In the case of cationic, nonionic, and zwitterionic surfactants, whether the length of hydrophobic microblock plays a role in the rheological behavior should be further studied.

3.5. Fluorescence Investigation. The intensity ratio $I_{3} / I_{1}$ of the third $(385 \mathrm{~nm})$ to the first $(375 \mathrm{~nm})$ vibronic peak in the fluorescence emission spectrum of pyrene is sensitive to the polarity of the local microenvironment of the pyrene probe $[12,14]$. This ratio is directly proportional to polarity. Thus,

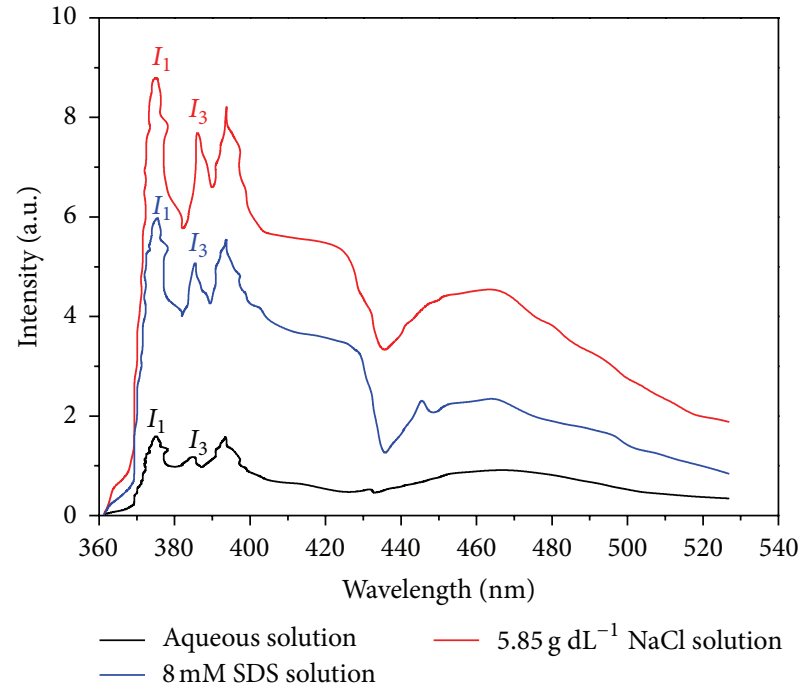

FIGURE 13: Fluorescence spectra of pyrene in $0.25 \mathrm{~g} \mathrm{dL}^{-1} 0.75 \mathrm{DM} 12.8$ solutions at different conditions.

the values of $I_{3} / I_{1}$ provide significant evidence for the formation of hydrophobic microdomains at salt concentrations and the development of bridge points or mixed micelles when surfactant is added. Figure 13 depicts the fluorescence spectra of $0.2 \mathrm{~g} \mathrm{dL}^{-1} 0.75 \mathrm{DM} 12.8$ for pyrene at different conditions. The $I_{3} / I_{1}$ value is 0.706 in aqueous solution, which increases to 0.835 in $8 \mathrm{mM}$ SDS solution where the pyrene probe is located in the mixed micelles constructed by surfactant molecules and hydrophobic segments of 0.75DM12.8. Additionally, the $I_{3} / I_{1}$ value in $5.85 \mathrm{~g} \mathrm{dL}^{-1}$ solution is 0.883 higher than that in aqueous solution, indicating that the hydrophobic microdomains became more compact in the high salt concentration. These results agree favorably with that obtained from viscosity.

3.6. Morphology of Networks. The microscopic structures of poly(AM/DMDCC) at different experimental conditions are observed through ESEM at room temperature and the morphological images are shown in Figure 14. In extremely dilute solution of copolymer 0.5DM12.7, intramolecular associations of hydrophobic segments compact polymers chains and an irregular microstructure is observed, which, of course, lowers viscosity. At the critical association concentration shown in Figure 14(b), three-dimensional network formed by intermolecular association results in a regular morphology and dramatically enhances solution viscosity. These three-dimensional networks are disrupted in the presence of higher concentration salt and broke into small clusters. As shown in Figure 14(c), for copolymer 0.75DM12.8, morphology of salt aggregation is observed due to high concentration of salt. At higher polymer concentration, as exhibited in Figure 14(d), high degree of chain entanglement protects three-dimensional networks from disruption and keeps viscosity relatively stable. Adding SDS, a significantly cooperative interaction between the copolymer chain and 


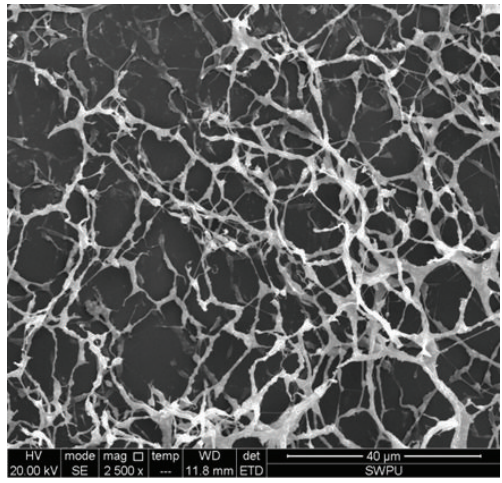

(a)

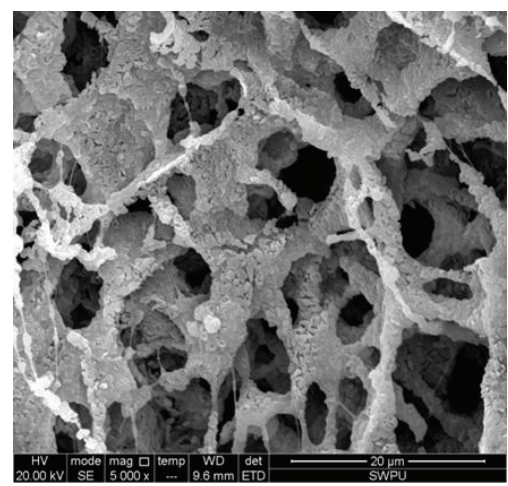

(d)

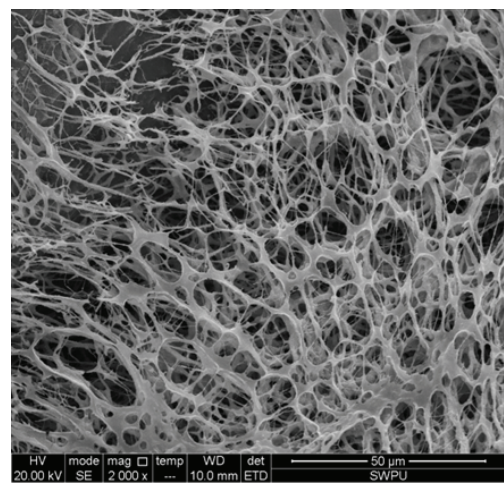

(b)

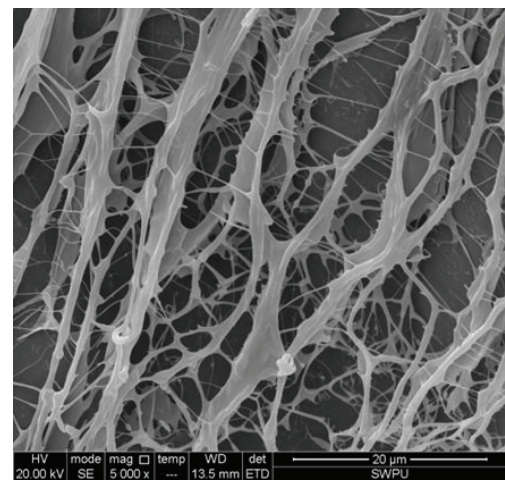

(e)

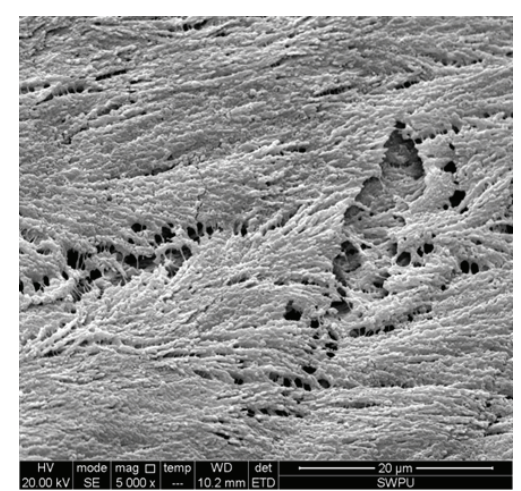

(c)

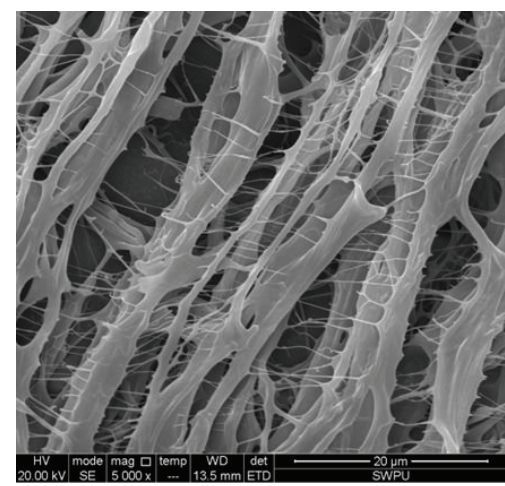

(f)

FIGURE 14: ESEM images of poly(AM/DMDCC) at different experimental conditions; (a) 0.5DM12.7 with 0.075 g dL ${ }^{-1}$; (b) $0.5 \mathrm{DM} 12.7$ with $0.15 \mathrm{~g} \mathrm{dL}^{-1}$; (c) $0.75 \mathrm{DM} 12.8$ with $0.15 \mathrm{~g} \mathrm{dL}^{-1}$ in $2 \mathrm{~g} \mathrm{dL}^{-1}$ salt solution; (d) $0.75 \mathrm{DM} 12.8$ with $0.75 \mathrm{~g} \mathrm{dL}^{-1}$ in $2 \mathrm{~g} \mathrm{dL}^{-1}$ salt solution; (e) $0.5 \mathrm{DM} 12.7$ with $0.5 \mathrm{~g} \mathrm{dL}^{-1}$ in $8 \mathrm{mM}$ SDS solution; (f) $0.75 \mathrm{DM} 12.8$ with $0.5 \mathrm{~g} \mathrm{dL}^{-1}$ in $8 \mathrm{mM}$ SDS solution.

surfactant leads to more regular microstructures than these of copolymer solution. Moreover, for copolymer 0.75DM12.8 with the longest $N_{H}$, at the CMC of SDS, both intermolecular hydrophobic interactions and ionic attractions are cooperative to promote the aggregation behavior of copolymer; thus, the most regular microstructures are observed.

\section{Conclusion}

A novel cationic surfmer, methacryloxyethyl-dimethyl cetyl ammonium chloride (DMDCC), was synthesized in the experiment. For DMDCC-SDS binary mixed surfactants system, conductivity measurement and a steady-state fluorescence technique indicated that synergetic mixed micelles were obtained due to strong net attraction between cationic (DMDCC) and anionic (SDS) surfactants, which dramatically reduce the CMC and keep the Nagg almost stable. Therefore, this new method could not only reasonably adjust the length of the hydrophobic microblock $\left(N_{H}\right)$ but also sharply reduce the amount of SDS. Utilizing a DMDCC-SDS mixed micellar copolymerization method, a series of watersoluble associative copolymers with acrylamide (AM) and DMDCC are synthesized.

The incorporation of hydrophobic segments into polymer chains induces a strong enhancement of apparent viscosity.
Rheological measurements including steady flow and linear viscoelasticity experiments are used to study the association process in the semidilute regime of copolymers. In the whole shear rate range of steady flow experiments, a shear thickening behavior of copolymers is exhibited and followed by shear thinning. Increasing hydrophobic microblock enhances the degree of association and results in shear thickening behavior towards lower share rate. The presence of the hydrophobic group into macromolecular chain increased both storage $\left(G^{\prime}\right)$ and loss $\left(G^{\prime}\right)$ modulus. At low frequencies, solution behaves as a Newtonian liquid and, at high frequencies, originating from strong intermolecular association, solution exhibits as an elastic solid with storage modulus higher than loss modulus. Relaxation time $\left(t_{c}\right)$ is also calculated to interpret the degree of intermolecular association formed by hydrophobic segments. And the results indicate that both the hydrophobe content and especially the length of the hydrophobic microblock are found to be the two main factors inducing an increase in the final relaxation time.

In the vicinity of the critical associative concentration, the presence of salt influences the dynamics transformation between intramolecular and intermolecular junctions resulting in changing the solution viscosity. However, at higher copolymer concentration, governed by chains entanglement and intermolecular associations, viscosity enhancement is 
observed in the whole salt concentration range. Apparent viscosity measurement performed on the copolymer indicates that both ionic attraction and intermolecular interactions play a great role. At the CMC of SDS, the formation of mixed micelles between the copolymer chain and surfactant which serve as junction bridges for transitional network remarkably enhances the viscosity. What is more, the microscopic structures for copolymers at different experimental conditions conducted by ESEM confirm the results discussed above.

\section{Conflict of Interests}

The authors declare that there is no conflict of interests regarding the publication of this paper.

\section{Acknowledgment}

The authors are grateful to the Major Special Project of China (Grant no. 20082X05049-05-03) for financial support of this work.

\section{References}

[1] A. Hill, F. Candau, and J. Selb, "Properties of hydrophobically associating polyacrylamides: influence of the method of synthesis," Macromolecules, vol. 26, no. 17, pp. 4521-4532, 1993.

[2] E. Volpert, J. Selb, and F. Candau, "Associating behaviour of polyacrylamides hydrophobically modified with dihexylacrylamide," Polymer, vol. 39, no. 5, pp. 1025-1033, 1998.

[3] S. Wu, R. A. Shanks, and G. Bryant, "Properties of hydrophobically modified polyacrylamide with low molecular weight and interaction with surfactant in aqueous solution," Journal of Applied Polymer Science, vol. 100, no. 6, pp. 4348-4360, 2006.

[4] M. Camail, A. Margaillan, and I. Martin, "Copolymers of Nalkyl-and N-arylalkylacrylamides with acrylamide: influence of hydrophobic structure on associative properties. Part I: viscometric behaviour in dilute solution and drag reduction performance," Polymer International, vol. 58, no. 2, pp. 149-154, 2009.

[5] Q. Yang, C. Song, Q. Chen, P. Zhang, and P. Wang, "Synthesis and aqueous solution properties of hydrophobically modified anionic acrylamide copolymers," Journal of Polymer Science B: Polymer Physics, vol. 46, no. 22, pp. 2465-2474, 2008.

[6] M. S. Kamal, I. A. Hussien, A. S. Sultan, and M. Han, "Rheological study on ATBS-AM copolymer-surfactant system in high-temperature and high-salinity environment," Journal of Chemistry, vol. 2013, Article ID 801570, 9 pages, 2013.

[7] P. Zhang, Y. Wang, W. Chen, H. Yu, Z. Qi, and K. Li, "Preparation and solution characteristics of a novel hydrophobically associating terpolymer for enhanced oil recovery," Journal of Solution Chemistry, vol. 40, no. 3, pp. 447-457, 2011.

[8] C. Zhong, P. Luo, Z. Ye, and H. Chen, "Characterization and solution properties of a novel water-soluble terpolymer for enhanced oil recovery," Polymer Bulletin, vol. 62, no. 1, pp. 7989, 2009.

[9] F. Liu, M. Yao, and M. T. Run, "Synthesis and characterizations of poly (trimethylene terephthalate)-b-poly (tetramethylene glycol) copolymers," International Journal of Polymer Science, vol. 2013, Article ID 156289, 10 pages, 2013.
[10] U. P. Strauss and B. L. Williams, "The transition from typical polyelectrolyte to polysoap. III. Light scattering and viscosity studies of poly-4-vinylpyridine derivatives," The Journal of Physical Chemistry, vol. 65, no. 8, pp. 1390-1395, 1961.

[11] R. Varoqui and U. P. Strauss, "Comparison of electrical transport properties of anionic polyelectrolytes and polysoaps," The Journal of Physical Chemistry, vol. 72, no. 7, pp. 2507-2511, 1968.

[12] C. L. McCormick, C. E. Hoyle, and M. D. Clark, "Water-soluble copolymers. 35. Photophysical and rheological studies of the copolymer of methacrylic acid with 2-(1-naphthylacetyl)ethyl acrylate," Macromolecules, vol. 23, no. 12, pp. 3124-3129, 1990.

[13] Y. Morishima, T. Kobayashi, T. Furui, and S.-I. Nozakura, "Intramolecular compartmentalization of photoredox centers in functionalized amphiphilic polyelectrolytes: a model for collisionless electron transfer systems," Macromolecules, vol. 20, no. 7, pp. 1707-1712, 1987.

[14] M. A. Winnik, S. M. Bystryak, and J. Siddiqui, "Interaction of pyrene-labeled poly(ethylene imine) with sodium dodecyl sulfate in aqueous solution," Macromolecules, vol. 32, no. 3, pp. 624-632, 1999.

[15] B. Gao, H. Guo, J. Wang, and Y. Zhang, "Preparation of hydrophobic association polyacrylamide in a new micellar copolymerization system and its hydrophobically associative property," Macromolecules, vol. 41, no. 8, pp. 2890-2897, 2008.

[16] E. Volpert, J. Selb, and F. Candau, "Influence of the hydrophobe structure on composition, microstructure, and rheology in associating polyacrylamides prepared by micellar copolymerization," Macromolecules, vol. 29, no. 5, pp. 1452-1463, 1996.

[17] A. Samakande, P. C. Hartmann, and R. D. Sanderson, "Synthesis and characterization of new cationic quaternary ammonium polymerizable surfactants," Journal of Colloid and Interface Science, vol. 296, no. 1, pp. 316-323, 2006.

[18] T. Noda, A. Hashidzume, and Y. Morishima, "Solution properties of micelle networks formed by nonionic surfactant moieties covalently bound to a polyelectrolyte: salt effects on rheological behavior," Langmuir, vol. 16, no. 12, pp. 5324-5332, 2000.

[19] A. B. Lowe and C. L. McCormick, "Synthesis and solution properties of zwitterionic polymers," Chemical Reviews, vol. 102, no. 11, pp. 4177-4189, 2002.

[20] C. Thévenot, B. Grassl, G. Bastiat, and W. Binana, "Aggregation number and critical micellar concentration of surfactant determined by Time-Dependent Static Light Scattering (TDSLS) and conductivity," Colloids and Surfaces A: Physicochemical and Engineering Aspects, vol. 252, no. 2-3, pp. 105-111, 2005.

[21] A. A. Dar, G. M. Rather, and A. R. Das, "Mixed micelle formation and solubilization behavior toward polycyclic aromatic hydrocarbons of binary and ternary cationic-nonionic surfactant mixtures," The Journal of Physical Chemistry B, vol. 111, no. 12, pp. 3122-3132, 2007.

[22] B. Jönsson, B. Kronberg, and B. Lindman, Surfactants and Polymers in Aqueous Solution, John Wiley \& Sons, 2003.

[23] X. F. Zhang and W. H. Wu, "Novel hydrophobically associative polyacrylamide with tunable viscosity," Chinese Chemical Letters, vol. 20, no. 11, pp. 1361-1365, 2009.

[24] D. Attwood, V. Mosquera, J. Rodriguez, M. Garcia, and M. J. Suarez, "Effect of alcohols on the micellar properties in aqueous solution of alkyltrimethylammonium bromides," Colloid \& Polymer Science, vol. 272, no. 5, pp. 584-591, 1994.

[25] J. X. Liu, Y. J. Guo, Y. W. Zhu et al., "Polymerization of microblock associative polymer with alcohol-surfmer mixed micellar method and their rheological properties," Acta Physico-Chimica Sinica, vol. 28, no. 7, pp. 1757-1763, 2012. 
[26] W. Jaeger, J. Bohrisch, and A. Laschewsky, "Synthetic polymers with quaternary nitrogen atoms-synthesis and structure of the most used type of cationic polyelectrolytes," Progress in Polymer Science, vol. 35, no. 5, pp. 511-577, 2010.

[27] A. Kotzev, A. Laschewsky, P. Adriaensens, and J. Gelan, "Micellar polymers with hydrocarbon and fluorocarbon hydrophobic chains. A strategy to multicompartment micelles," Macromolecules, vol. 35, no. 3, pp. 1091-1101, 2002.

[28] Y. Chang and C. L. McCormick, "Water-soluble copolymers. 49. Effect of the distribution of the hydrophobic cationic monomer dimethyldodecyl(2-acrylamidoethyl)ammonium bromide on the solution behavior of associating acrylamide copolymers," Macromolecules, vol. 26, no. 22, pp. 6121-6126, 1993.

[29] K. Stähler, J. Selb, and F. Candau, "Multicompartment polymeric micelles based on hydrocarbon and fluorocarbon polymerizable surfactants," Langmuir, vol. 15, no. 22, pp. 7565-7576, 1999.

[30] E. J. Regalado, J. Selb, and F. Candau, "Viscoelastic behavior of semidilute solutions of multisticker polymer chains," Macromolecules, vol. 32, no. 25, pp. 8580-8588, 1999.

[31] C. Zhong, R. Huang, L. Ye, and H. Dai, "Study on the morphology of the novel hydrophobically associative acrylamidebased terpolymer in aqueous solution by AFM measurements," Journal of Applied Polymer Science, vol. 101, no. 6, pp. 39964002, 2006.

[32] L. Leibler, M. Rubinstein, and R. H. Colby, "Dynamics of reversible networks," Macromolecules, vol. 24, no. 16, pp. 47014707, 1991. 

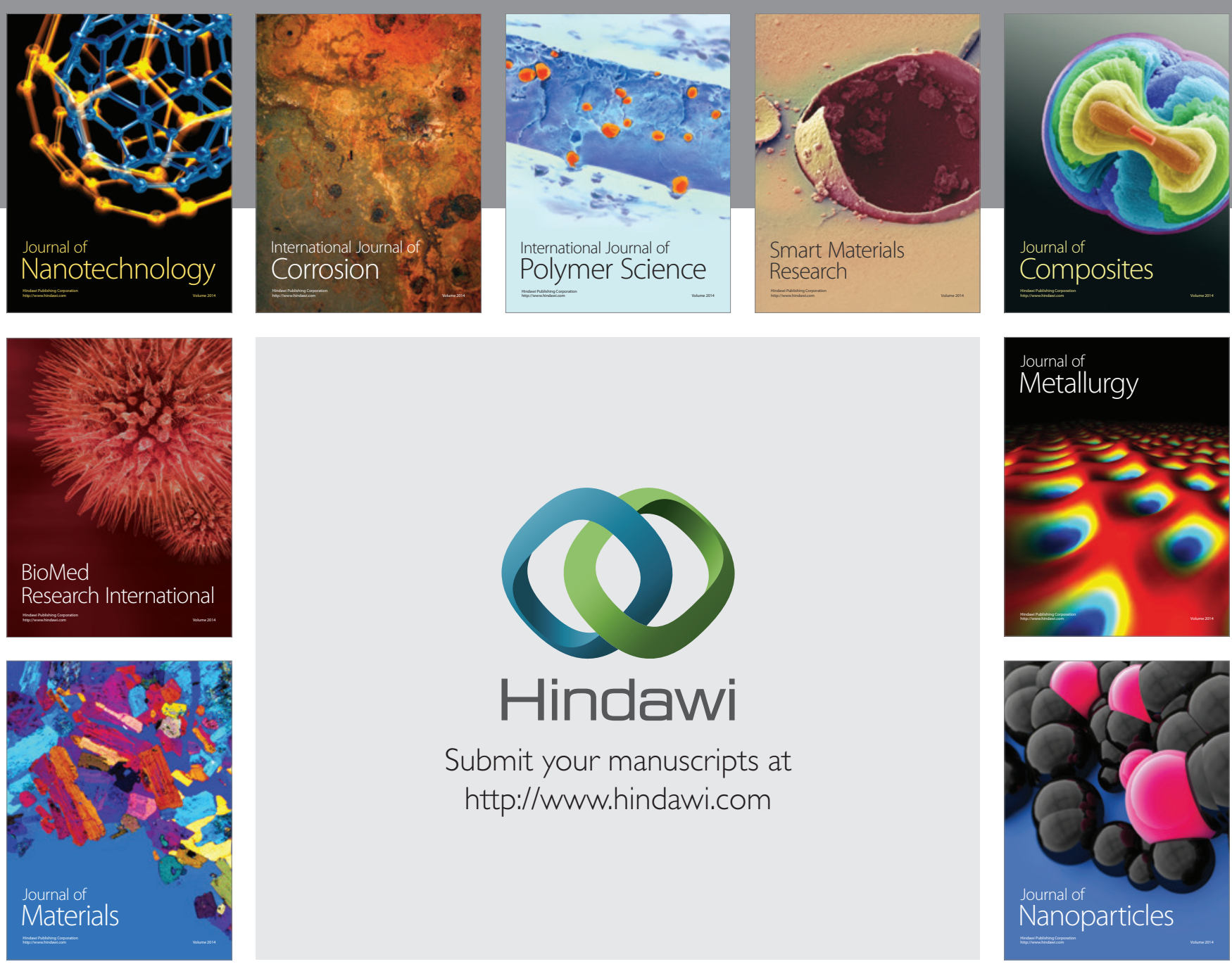

Submit your manuscripts at http://www.hindawi.com
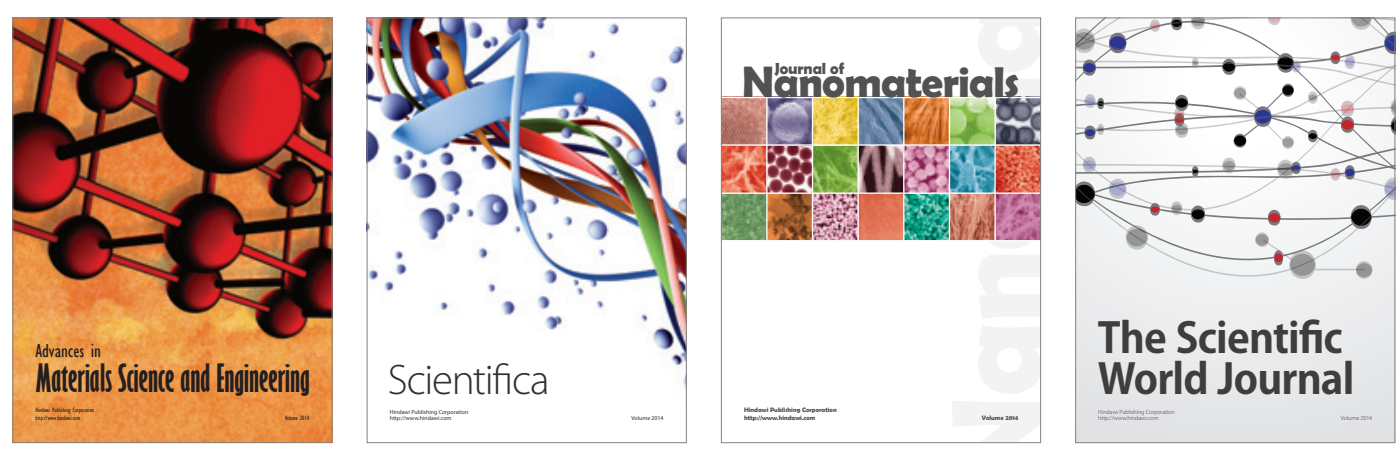

\section{The Scientific World Journal}
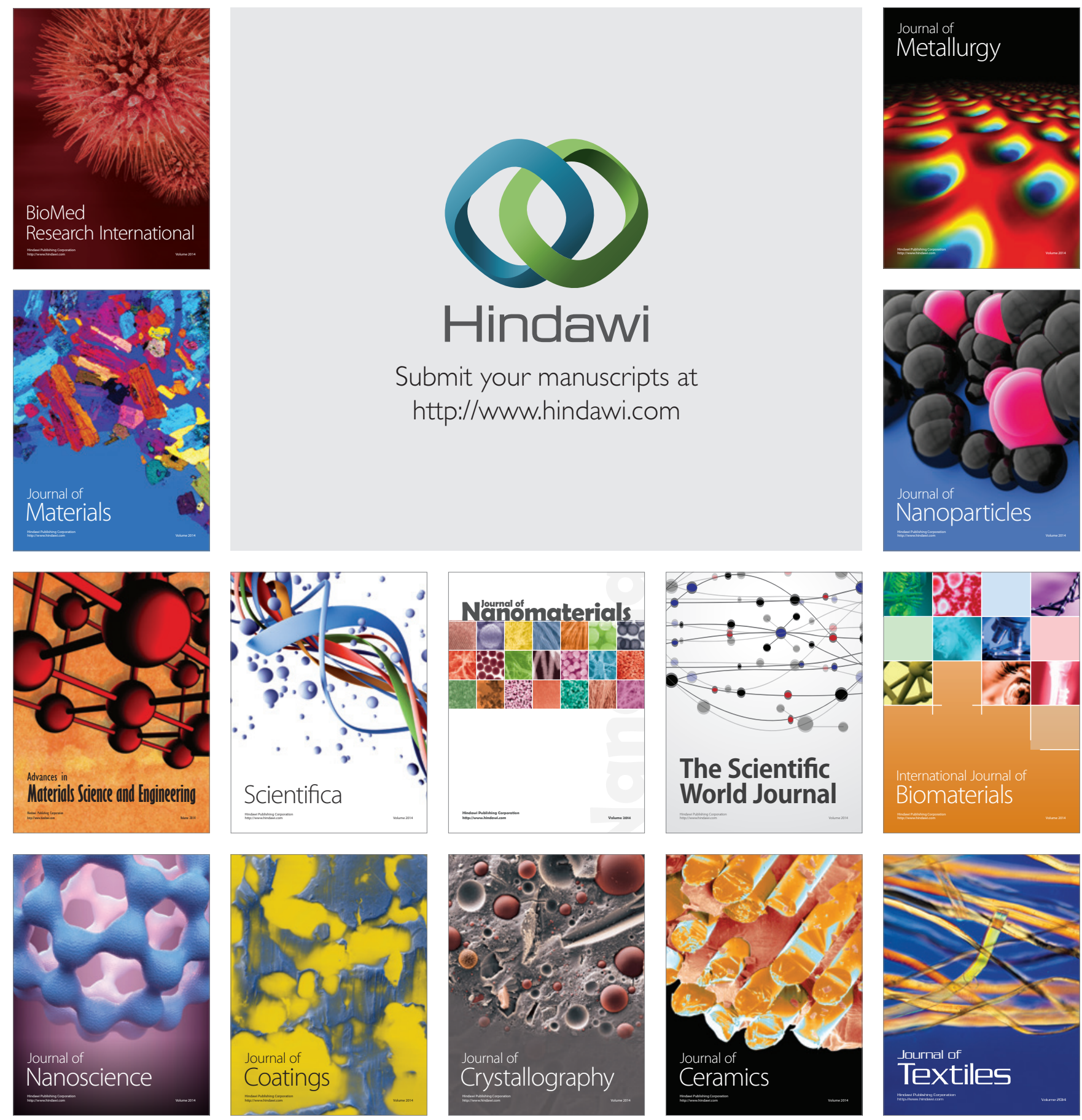